\title{
ASYMPTOTIC STATES FOR EQUATIONS OF REACTION AND DIFFUSION
}

BY PAUL C. FIFE ${ }^{1}$

\section{Contents}

1. Introduction

2. Asymptotic states

3. The scalar case: Fisher's equation

4. Systems: stationary solutions
(a) Small amplitude stationary solutions
(b) Larger amplitude solutions: peaks
(c) Larger amplitude solutions: asymptotic methods

5. Plane wave trains
(a) Small amplitude wave trains
(b) Wave extensions from homogeneous oscillations
(c) Wave trains in excitable media
(d) Wave trains for predator-prey equations

6. Plane wave fronts
(a) Gradient systems
(b) Small amplitude fronts
(c) Systems with small parameters
(d) A model from population genetics

7. Pulses

8. Targets and spirals; slow modulation

9. Close relatives of reaction-diffusion equations

(a) Discrete versions

(b) Integrodifference equations

(c) Integrodifferential equations in neurodynamics and epidemiology

References

1. Introduction. The term "equations of reaction and diffusion" is usually taken to mean semilinear systems of second order partial differential equations of the form

$$
\partial u / \partial t=D \Delta u+f(x, t, u, \nabla u), \quad u=\left(u_{1}, \ldots, u_{m}\right),
$$

or generalizations made by replacing the Laplace operator by linear or quasilinear elliptic operators. Here the "diffusion matrix" $D$ has nonnegative

An Invited Address delivered at the 746th meeting of the Society at Hayward, California, April 22, 1977; received.by the editors September 28, 1977.

AMS (MOS) subject classifications (1970). Primary 35K55, 35B40; Secondary 35B10, 35B25, 35Q99, 35R30.

'Supported by NSF Grant MCS77-02139. 
elements, and in most applications is diagonal.

My object here is to survey our knowledge of the "ultimate" or "steady state" behavior of solutions of (1.1), as opposed to their transient behavior (steady state does not mean time-independent; the word "stationary" will be reserved for the latter). Roughly speaking, what this means is that we will be seeking special solutions which are approached by many other solutions as $t \rightarrow \infty$. This idea will be made clearer in $\$ 2$, where we speak of stable asymptotic states.

But first, an indication of the importance of equations of this type. They occur widely as models for the dynamics of large multispecies populations whose individuals are capable of random spatial migration. In this context $x$ represents position in space ( $\Delta$ is the Laplacian in $x$ ), $t$ is time, and at least some of the components of $u$ represent spatial densities of the various species. In the prototypical example of this modeling, the species are chemical species, and the individuals of the population are molecules. Their random migration through the chemically reacting medium is called diffusion.

Of course (1.1) also appears as a model for problems outside of population or chemical dynamics, one important example being the nerve conduction models discussed in $\$ 7$.

For population problems, the term $D \Delta u$ is a convenient approximation, justifiable under various circumstances, for the rate of change of the population $u$ at any given position and time, due to random spatial migration. The term $f$ measures the rate of change due to "reaction processes" in the medium, such as chemical reactions, reproduction processes or deaths in a biological population, and material transfer across a membrane.

I shall occasionally touch upon the contexts in which the equations are studied; the reader can learn more about this by consulting the works listed in the bibliography. But my emphasis will be on properties of the solutions.

Attention will generally be confined to solutions defined for all $x \in \mathbf{R}^{n}$. In applications, however, one often seeks solutions of (1.1) in a bounded $x$-domain (the "medium"), and conditions them by processes assumed to occur on the boundary of this domain. Then the ultimate behavior of solutions will be affected by the boundary conditions as well as the internal processes, described by the equations (1.1) themselves. When the size of the medium is large or the diffusion matrix $D$ is small, then the behavior in the interior of the medium is typically little affected by the boundary conditions, and the determination of that behavior can be simplified by assuming the medium to occupy all space, as we do. For example, the effect of the boundary on a stationary solution is often confined to a boundary layer; and wave trains and pulses, though strictly defined only for an infinite medium, still reflect actually occurring phenomena in bounded ones. This, plus the finite length of this talk, will be my justification for ignoring boundary influences.

Reaction-diffusion systems (1.1) include two very important extreme cases:

(1) $D=0 ; f$ independent of $x$ and of $\nabla u$ :

$$
d u / d t=f(t, u) .
$$

This is called the system of "kinetic equations" associated with (1.1). 
(2) $m=1, f=0, D>0$ :

$$
\partial u / \partial t=D \Delta u \quad(u \text { scalar })
$$

This is the well-known scalar diffusion, or heat, equation.

To say that ordinary differential equations and diffusion equations have each been studied extensively in their own rights would be an understatement. Though both represent evolution processes, the two theories have relatively little in common. In some sense the theory of reaction-diffusion equations attempts to bridge the gap between them. (A thorough account of aspects of the theory of reaction-diffusion equations different from those emphasized here can be found in Henry (1978).) In pursuing this subject, an interesting question to keep in mind is, how does the presence of diffusion effects modify the rich dynamical theory available for the kinetic equations (1.2)? This point of view is relevant in studying wave fronts, which connect two rest states of (1.2), and wave trains, some of which modify periodic solutions of (1.2).

Even when $D$ is not $0,(1.2)$ serves as the equation satisfied by $x$-independent solutions of (1.1) (still assuming $f$ independent of $\nabla u$ ). For this reason, the dynamics, and in particular the asymptotic states, of (1.1) include those of (1.2). Despite the rich theory behind (1.2), we shall call $x$-independent dynamics "trivial" and concentrate on contrary cases-asymptotic states in which diffusion is important.

It might be well to summarize the restrictions under which we shall operate.

(1) We consider solutions defined for all $x \in \mathbf{R}^{n}$.

(2) Most of ten, we treat only a single spatial coordinate, $n=1$. When this restriction is made, the reason is not so much for simplicity of exposition as it is because in those contexts, not much is known for $n>1$.

(3) $f$ independent of $x, t$, and $\nabla u$, so $f=f(u)$. Work has been done on extending some of the results listed here to inhomogeneous media (for example, see Ortoleva and Ross (1974)).

(4) We exclude consideration of asymptotic states independent of $x$.

Under the heading "asymptotic behavior of solutions," two questions naturally arise:

(1) What are the possible long-time behaviors of solutions?

(2) Given the initial data $u(x, 0)$ of a particular solution, what is its long-time behavior?

This talk will be mainly concerned with only the first of these difficult questions. With the exception of scalar equations, known results under the second category mainly relate to $x$-independent asymptotic states in bounded domains, which I have decided not to discuss. The most definitive results here are probably those of Conway, Hoff, and Smoller (1978).

The first question can be answered relatively completely in the case of a scalar nonlinear diffusion equation. The situation for this case is outlined in \$3. \$\$4-8 describe most of the presently known results bearing on this same question, for systems with $m>1$. A far greater variety of asymptotic states is possible for $m>1$, though it is by no means true that anything is possible. At the same time, a complete asymptotic state theory for systems is nowhere in sight, and so the existing results, varied though they may be, are still relatively 
meager. Rigorous results are truly meager. For example, the only known proofs of $C_{0}$-stability of nontrivial asymptotic states for systems with $m>1$ are the one for stationary peaks referred to in $\$ 4(b)$, and the one for pulse solutions of a piecewise linear nerve conduction equation discussed in \$7. (In addition, linear stability criteria have been verified rigorously for some types of wave trains-see $\$ 5(\mathrm{~b})$.)

Asymptotic states in various categories are treated in \$\$4-8: stationary states, wave trains, wave fronts, pulses, centers, and other forms.

In modeling natural phenomena, various researchers have devised evolution equations which are not partial differential equations, but whose solutions have the essential features of those of reaction-diffusion systems. These equations are deserving of mathematical theories, and some results have been established. In $\$ 9$ a few of these models will be reviewed briefly.

The survey nature of the talk means, of course, that I shall feel free to omit details at will. In fact, most often I shall be content with merely stating a result and giving a bare indication of the method behind it. When the results and procedures are not so easily available in the literature, however, I shall often expand on them.

Other aspects of nonlinear diffusion, as well as more details concerning some topics covered here, can be found in the readable monograph compiled by Diekmann and Temme (1976).

2. Asymptotic states. Considering functions $f$ which depend only on $u$, we rewrite (1.1) as

$$
\partial u / \partial t=D \Delta u+f(u) .
$$

We shall be concerned with those solutions of (2.1) which exist for all $t>t_{0}$, for some finite $t_{0}$, and this property of solutions will be assumed without further mention. Initial value problems, say with bounded piecewise continous initial data, always have local solutions, and criteria on the equation and the initial data have been given by various people under which a global solution is assured. The most complete results of this sort are probably those of Amann (1978).

Given a particular reaction-diffusion system or class of systems, a natural goal is to determine all possible stable long-time behaviors of its solutions. At present this is a realistic goal only for restrictive examples, such as scalar equations ( $\$ 3), \lambda-\omega$ systems ( $\$ 5)$, and piecewise linear systems, such as McKean's simplification of the nerve conduction equations (\$7), all of this in one space variable. Nevertheless, continual progress in this direction is being achieved for more general classes of systems as well, as I hope to bring out in this paper.

We need to say what is meant by long-term behavior, and we shall define it as an equivalence class of solutions which are comparable in a certain sense as $t \rightarrow \infty$. In comparing solutions, it really should not matter if one is displaced from another in space or time. More generally, let $G$ be the group of transformations on $(x, t)$ generated by rigid motions and reflections in $x$, and translations in $t$. Clearly these transformations leave the set of solutions of (2.1) invariant. If $T \in G$, we note by $T u$ the solution obtained from $u$ by 
operating on $(x, t)$ by the transformation $T$. We shall consider $u$ and $T u$ to be equivalent. For particular systems (2.1) with added symmetry properties, the group $G$ can be extended, perhaps, to act on the vector $u$ as well. In such cases, it may be desirable to use the larger group in defining equivalence. On the other hand for problems in which $f$ depends on $\nabla u, G$ may have to be reduced to the group of $(x, t)$-translations.

Definition. Let $u_{1}, u_{2}$ be two solutions of (2.1) for all $x$ and for large enough $t$. They are asymptotically equivalent if, for some $T \in G$,

$$
\lim _{t \rightarrow \infty} \sup _{x}\left|u_{1}(x, t)-T u_{2}(x, t)\right|=0 .
$$

DefinImon. An asymptotic state for (2.1) is an (asymptotic) equivalence class of solutions.

The class asymptotically equivalent to a given $u$ is denoted by [ $u$ ]. If $\phi$ is a bounded continuous function of $x$, we denote by $u_{\phi}$ the solution of the initial value problem with initial data $\phi$.

We take the stance that the stable asymptotic states (SAS's) are the important ones, as they are the ones generally seen in applied contexts. The symbol $C_{0}\left(\mathbf{R}^{n}\right)$ will denote the space of continuous functions of $x \in \mathbf{R}^{n}$ with norm $\|\phi\|=\sup _{x}|\phi(x)|$.

Definition. An asymptotic state $[u]$ is stable if the set

$$
\left\{\phi: u_{\phi} \in[u]\right\}
$$

is open in $C_{0}\left(\mathbf{R}^{n}\right)$.

In the following, we call this $C_{0}$-stability.

Implicit in this definition is that for $\phi$ in this set, $u_{\phi}$ exists globally. This set is nonempty; in fact it contains the functions $u(\cdot, t)$ for all large enough $t$. SAS's may be represented by solutions which can be perturbed by a uniformly small function without destroying their longtime behavior.

In the case of stationary or plane wave solutions, another definition for stability is in terms of the $L_{\infty}$ spectrum of the linearization of the right side of (2.1) about the solution in question (the problem should be cast in a moving coordinate frame in the case of plane waves). The criterion is that except for a simple eigenvalue at the origin (which always exists), the spectrum is in the left half plane and bounded away from the imaginary axis. Sattinger (1977a, b; 1977a) proved that in typical cases, stability in this sense implies $C_{0}$-stability.

When looking for SAS's for particular systems (2.1), it is natural to focus attention on special classes of them. One particular class consists of those independent of $x$, hence solutions of the corresponding kinetic equation; we have called them "trivial." Those which depend on $x$ may be called "dissipative structures," a term introduced by Prigogine and coworkers (Prigogine and Nicolis (1967)).

Another important class of asymptotic states are those of permanent type, meaning that some representative exists, as a solution of (2.1) for all $t \in \mathbf{R}$, not just for large enough $t$. Subclasses of asymptotic states are those with a representative in one of the following categories:

Stationary $(t$-independent) solutions, 
Solutions periodic in $t$,

Traveling waves: $u(x, t)=V(x-c t)$ for some velocity vector $c$,

Plane waves: traveling waves with $V(z)=U(z \cdot \nu)$ for some vector $\nu$ (i.e., $u=U(x \cdot \nu-c t), c$ a scalar),

Wave trains ( $U$ periodic),

Wave fronts $(U(-\infty)$ and $U(\infty)$ exist and are unequal),

Pulses $(U( \pm \infty)$ exist and are equal; $U$ not constant).

Other forms which have been studied are target patterns $(u(x, t)=$ $U(|x|, t), U$ periodic in $t)$, rotating spiral patterns $(n=2, x=(r \cos \theta, r$ $\sin \theta), u(x, t)=U(r, \theta-c t), U$ periodic in second argument), and expanding circular or spherical fronts. The latter are not of permanent type.

Problem. Determine the nontrivial SAS's of the above types.

The aim of the paper is to outline most of the known partial solutions to this problem.

3. The scalar case: Fisher's equation. The scalar nonlinear differential equation

$$
u_{t}=u_{x x}+f(u)
$$

arises in many applications. The particular case when $f=m u(1-u)$ (the logistic equation with diffusion added) was introduced by Fisher (1937) in connection with "genetic waves." These are simply wave front solutions; and since fronts generally exist when $f$ has two or more zeros, equation (3.1) with this property is sometimes referred to as "Fisher's nonlinear diffusion equation."

The corresponding kinetic equation

$$
u^{\prime}=f(u)
$$

has very simple dynamics; they are determined by the zeros of $f$ and its signs in the intervening intervals. The zeros and signs of $f$ also determine the possible stable asymptotic states of (3.1).

First, we examine the case when $f$ has exactly one zero, at $u=u_{0}$, and it is stable with reference to (3.2). This means $f\left(u_{0}\right)=0, f(u)>0$ for $u<u_{0}$, $f(u)<0$ for $u>u_{0}$. Then (3.2) trivially has only a single SAS [ $\left.u_{0}\right]$. As it turns out, (3.1) likewise has $\left[u_{0}\right]$ as its only bounded SAS. This latter is proved by a simple comparison argument, using the fact that every bounded solution of (3.1) has solutions of (3.2) as sub- and super-solutions (see, for example, Aronson and Weinberger (1975)).

Suppose now that $f$ has a single zero, $u_{0}$, and it is unstable, so that $f$ changes sign from negative to positive as $u$ increases through $u_{0}$. It can be shown that (3.1) has an infinite number of bounded stationary solutions, all of them periodic in $x$. It further turns out that all of them are unstable (Fife (1977a)). The proof of this fact is again based on a comparison principle given by Aronson and Weinberger (1975).

CONJECTURE. If $f$ has a single zero and it is an unstable rest state of (3.2), then (3.1) has no bounded stable asymptotic states.

The instability result mentioned above shows that it has no bounded stable stationary states; the conjecture claims the nonexistence of time-dependent 
ones as well. The claim should be within range of a proof; in fact the corresponding result for a bounded $x$-interval with no-flux boundary conditions may be proved with the aid of a Lyapunov function technique.

Next, consider the case when $f$ has exactly two zeros, each of them simple. Then as rest states of (3.2), one of the zeros will be stable and the other unstable. The former will also be a (trivial) SAS of (3.1), and our conjecture is that it is the only bounded one.

But now there are other asymptotic states which, though unstable in our sense, nevertheless are significant in some contexts. Suppose the two zeros of $f$ are 0 and 1 with, for example, $f>0$ for $u \in(0,1)$. Then 0 is an unstable rest state of both (3.1) and (3.2), whereas 1 is a stable rest state of them both. If $f^{\prime}(0)>0, f^{\prime}(1)<0$, there exist wave front solutions $u=U(x-c t)$, with $U(-\infty)=1, U(\infty)=0$. In fact (Kolmogorov, Petrovskii and Piskunov (1937); Aronson and Weinberger (1978)) there is a number $c^{*}>0$ such that for every value of the velocity $c \geqslant c^{*}$, a unique such wave front exists. Since these fronts have been studied extensively, it may be worthwhile mentioning their conceptual connection with population problems. For particular kinds of functions $f$ having the given properties, (3.1) has been used as a model in population genetics (Fisher (1937); Kolmogorov, Petrovskii and Piskunov (1937); Aronson and Weinberger (1975); plus many more papers in which $f$ depends on $x$ ). In these models, $u$ represents the frequency of an advantageous allele in a population, so should be restricted to the interval $[0,1]$. The model is highly idealized, but if we assume it may be qualitatively significant, the wave front of minimal speed $c^{*}$ would correspond to the later stages of the following "take-over" process. Imagine a population with none of the advantageous alleles present: then $u \equiv 0$. Suppose that at some instant of time, some individuals with the advantageous gene are introduced at one locality, either by mutation or from an outside source. The "zero" state being unstable, $u$ will increase and approach 1 uniformly on bounded $x$-intervals. Ultimately, the "one" state will presumably take over by means of a pair of fronts moving in opposite directions, each approaching in profile the front with speed $c^{*}$, and each with speed approaching $c^{*}$. This configuration is unstable in the sense we have defined it, but in the framework of population genetics may be meaningful, because the type of perturbation (mutation, introduction of foreign elements) needed to change it would be very rare. Furthermore, it is possible that these waves are stable in certain weighted $L_{\infty}$ norms (Sattinger (1976b, 1977a)). The results of Rothe (1975, 1978), Hoppensteadt (1975), Stokes (1976), Kametaka (1976), Uchiyama (1977), and McKean (1970) are also relevant here.

It should also be mentioned that most contemporary population genetic modeling with Fisher-type equations is in connection with the analysis of clines (nonconstant stationary solutions) when $f$ is allowed to depend on $x$. This is another story, which we shall not touch upon.

The final case we consider is when $f$ has exactly three zeros, the two outer ones stable, the inner one unstable, and all three simple. This also occurs as a population genetic model, as well as in the study of transmission lines, combustion theory, and some degenerate cases of nerve signal propagation. Assume the two stable zeros are at $u=0$ and 1 , and the unstable one lies in 
between, as shown in Figure 1 below.

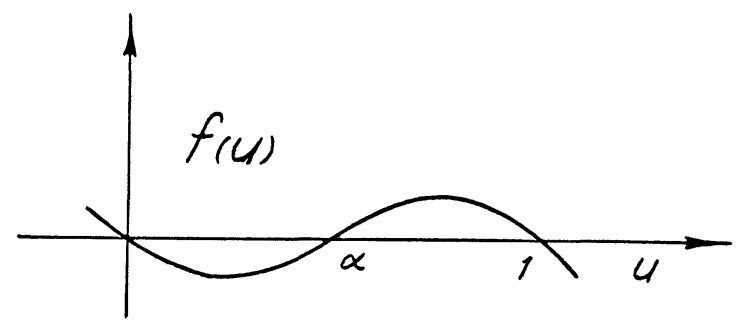

Figure 1

Clearly, the kinetic equation (3.2) and the original equation (3.1) have [0] and [1] as trivial stable asymptotic states. Now, for the first time, we find there are others as well. In fact, there turn out to be an infinite number of nontrivial stable asymptotic states, only two of which are interesting:

(1) There is a wave front (Kanel' (1962); Aronson and Weinberger (1975, 1978)) which can be thought of as connecting the two trivial stable rest states:

$$
u_{1}(x, t)=U(x-c t), \quad U(-\infty)=0, \quad U(\infty)=1 .
$$

It has a unique velocity $c$, and except for translation, a unique wave form as well. Its global stability was proved by Fife and McLeod (1977); see also Sattinger (1976b, 1977a) for local stability, and Chueh (1975) for a related result.

Reflection in $x$ yields a wave front traveling in the opposite direction: $u_{1}(-x, t)=U(-x-c t)$, which by our definition is equivalent to the former.

(2) If $c \neq 0$, a combination of $u_{1}$ and its reflection yields a second stable asymptotic state in the form of a diverging structure, as shown in Figure 2.

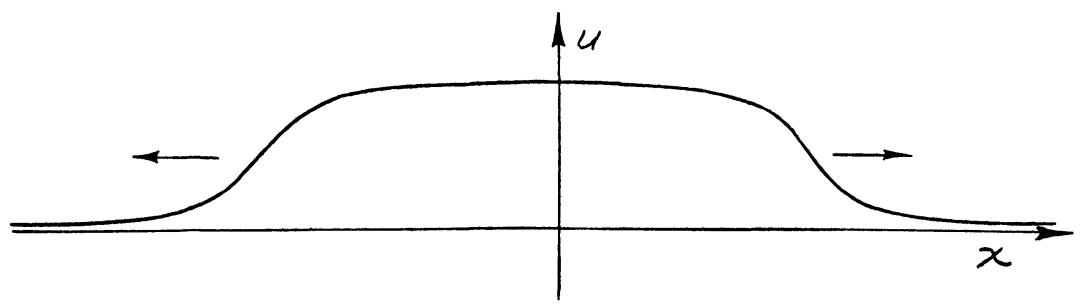

Figure 2

This asymptotic state may be represented by the function

$$
w(x, t)= \begin{cases}u_{1}(x, t), & c x \geqslant 0 \\ u_{1}(-x, t), & c x \leqslant 0\end{cases}
$$

even though $w$ itself is not an exact solution of (3.1). Its global stability was proved by Fife and McLeod (1977). Note that $[w]$ is not of permanent type, whereas $\left[u_{1}\right]$ is.

So we have two (one, if $c=0$ ) SAS's, $\left[u_{1}\right]$ and $[w]$, the first of permanent type. Other, rather artificial, SAS's can be constructed at will by combining an infinite number of wave fronts like the above, spaced at ever increasing distances apart. 
It should also be noted that there is an infinite number of unstable asymptotic states of (3.1) in this case, namely the periodic and other nonmonotone stationary ones (Fife (1977a)).

The following conjectures are probably true, and within range of proofs. If they can be proved, then one could say with a bit of justification that the $C_{0}$-asymptotic theory of Fisher's equation in one space dimension with three or fewer zeros is fairly complete:

CONJECTURE $1 .\left[u_{1}\right]$ is the only nontrivial stable asymptotic state of permanent type.

Let $\alpha \in(0,1)$ be the intermediate (unstable) zero of $f$.

CONJECTURE 2. If $0 \leqslant \phi \leqslant 1$ and $\phi$ is bounded away from $\alpha$ for large $|x|$, then $u_{\phi} \in[0],[1],\left[u_{1}\right],[w]$, or an unstable asymptotic state.

The asymptotic theory for Fisher's equation is quite simple when $f$ has fewer than three zeros, and more interesting but not too complex when the number is three, as above. If the number is more than three, then it is not too hard to envisage possible asymptotic states built by combining elementary wave fronts of the above type (elementary ones being those whose ranges span exactly three zeros of $f$ ). Some discussion of this was given by Fife and McLeod (1977). Probably all the stable ones can be found this way.

More difficult than the problem of finding the SAS's is the problem of determining their domains of attraction. In the case of Fisher's equation, partial characterizations of domains of attraction of the trivial stable states were given in Kanel' (1964), Chafee (1974), and Aronson and Weinberger (1975). The same was done for wave fronts in Kanel' (1962) and Fife and McLeod (1977). (See also the bibliography in the latter paper for results characterizing initial data which evolve to unstable wave fronts.)

An interesting generalization of the wave front problem for (3.1) was obtained by Larson (1977b). He considered the equation $u_{t}=u_{x x}+u_{y y}+$ $f(u)$ in a strip $0<y<1$ with zero Dirichlet conditions, and looked for traveling front solutions $u(x, y, t)=U(x-c t, y)$. Such fronts were obtained when $f(u)=m u(1-u)$ or is of a similar type.

4. Systems: stationary solutions. Existing work on nontrivial stable asymptotic states for systems gives ample evidence that they exist in great profusion and variety. Proved results in this direction, however, are relatively scarce; and at least for this reason, it is important to pay attention not only to results that have been established rigorously, but also to those obtained by heuristic, asymptotic, and computational methods. In this section, we survey most of what is known concerning nontrivial stable stationary solutions of systems. Most, but not all, of this body of knowledge concerns the case of one space variable. Therefore, we concentrate attention on the system

$$
u_{t}=D u_{x x}+f(u), \quad u=\left(u_{1}, \ldots, u_{m}\right),
$$

at times commenting on possible extensions to multidimensional problems.

First recall, from the previous section, that in the scalar case $m=1$ there are essentially no bounded stable stationary solutions, despite the fact that a great many unstable ones (mostly periodic in $x$ ) exist when $f$ has an unstable zero. Similar negative results hold for particular types of systems (Bardos and 
Smoller, (1978)). But in general, the situation is considerably different for systems, as we shall see.

(a) Small amplitude stationary solutions. Suppose $f$ vanishes at some value of $u$, which we take to be $0: f(0)=0$. Then in addition to the trivial solution $u \equiv 0$, it is sometimes possible to prove the existence of small amplitude solutions periodic in $x$. (Rigorous results of this type for problems on a finite interval were obtained by Auchmuty and Nicolis; see the survey by Auchmuty (1978).) The techniques are those of bifurcation theory. In this procedure, it is convenient to suppose an explicit dependence of the system (4.1) on a real parameter. In actual practice, models in the form of such systems practically always contain many adjustable parameters; for simplicity, we focus attention on a single one of them, denoted by $\lambda$. Typically, small amplitude nontrivial solutions may be constructed for $\lambda$ on a half-line $\left(\lambda>\lambda_{0}\right)$. On the basis of more standard bifurcation results, we again expect that in many cases some of the small amplitude nontrivial solutions near a critical point $\lambda_{0}$ will be stable, provided the trivial solution loses stability as $\lambda$ crosses $\lambda_{0}$. Thus the zero solution's stability may be transferred to a different type of solution. If the trivial solution is unstable for $\lambda$ in a full neighborhood of $\lambda_{0}$, then generally any bifurcating solution will also be unstable. (Segel and Jackson (1972) give an ecological interpretation.)

We explore the bifurcation of nontrivial solutions in the case when only the linear part of $f$ depends on $\lambda$ :

$$
f(u, \lambda)=(A+\lambda B) u+g(u)
$$

$g(u)=O\left(|u|^{2}\right)$ as $|u| \rightarrow 0$. Our results are mainly on the existence and multiplicity of such solutions; the question of transfer of stability has yet to be fully investigated.

The stability of constant solutions is most often tested by the "linearized criterion" described below, as it is easy to apply and rather intuitive. (To establish rigorously that the linearized criterion gives the correct answer for stability as we defined it in $\$ 2$ is a difficult matter, but has been done. The most complete results in this and related directions are those by Kielhöfer (1976).)

Consider the linearized system

$$
u_{t}=D u_{x x}+(A+\lambda B) u \text {. }
$$

For arbitrary real $k$, it has solutions of the form $u=\Phi e^{i k x+\mu}$ for any eigenvalue $\mu$ of the matrix $H\left(k^{2}, \lambda\right)=-k^{2} D+A+\lambda B$, with associated eigenvector $\Phi$. If, for all $k$, all eigenvalues $\mu$ have negative real part, then the zero solution is stable (linearized criterion); but if there is one with positive real part, for some $k$, it is unstable (l.c). The remaining case is that of marginal stability.

Assumption A. Let $\mu_{1}\left(k^{2}, \lambda\right)=\sigma\left(k^{2}, \lambda\right)+i \omega\left(k^{2}, \lambda\right)$ denote an eigenvalue of $H\left(k^{2}, \lambda\right)$ with maximal real part $\sigma$. Assume that for $(\lambda, k)$ in some rectangle $K$ with positive area, $\mu_{1}$ is algebraically simple, hence differentiable in $(\lambda, k)$, and is either real and unique or nonreal and unique except for its complex conjugate $\bar{\mu}_{1}$ (which must also be an eigenvalue since $H$ is real). Assume there is some $\left(\lambda_{0}, k_{0}\right)$ in the interior of $K$ with the properties 
(i) $\sigma\left(k_{0}^{2}, \lambda_{0}\right)=0$,

(ii) $\sigma\left(k^{2}, \lambda_{0}\right)<0$, for all $|k| \neq\left|k_{0}\right|$,

(iii) $\partial \sigma\left(k_{0}^{2}, \lambda_{0}\right) / \partial \lambda>0$.

Let $\omega_{0}=\omega\left(k_{0}^{2}, \lambda_{0}\right)$. There are two cases which can occur:

(I) $\omega_{0}=0$.

(II) $\omega_{0} \neq 0$.

It is easily seen that each case is generic. In this section we consider only case (I), reserving (II) for $\$ 5$.

THEOREM In case (I) there exists a two-parameter family $(u(x ; \varepsilon, k), \lambda(\varepsilon, k))$ of solutions of

$$
D u_{x x}+f(u, \lambda)=0
$$

defined and continuous in $(x, \varepsilon, k)$ for small $\varepsilon$ and $\left|k-k_{0}\right|$, such that

(i) $u(x ; \varepsilon, k)$ is even and (when $k \neq 0$ ) periodic in $x$ with wave number $k$;

(ii) $\operatorname{Max}\|u(x ; \varepsilon, \lambda)\|=\varepsilon$;

(iii) $\lambda\left(0, k_{0}\right)=\lambda_{0}$, and the range of the function $\lambda(\varepsilon, k)$ includes at least $a$ half-neighborhood of the point $\lambda_{0}$.

Thus, small solutions of arbitrary wave length close to $2 \pi / k_{0}$ are obtained. If $k_{0}=0$, then we obtain small solutions of sufficiently large wave length. The proof of this result is considerably more involved in the case $k_{0}=0$ than it is in the case $k_{0} \neq 0$. In the latter case, it is sketched in Fife (1977a). The solution to lowest order is sinusoidal in $x$.

In the case $k_{0}=0$, the solution to lowest order takes the form $u \approx \varepsilon \Phi a_{k}(x)$, where $\Phi$ is a constant vector (the eigenvector of $H\left(0, \lambda_{0}\right)$ ) and $a_{k}$ is a scalar periodic function with long wave length $2 \pi / k$, which is not sinusoidal. In the limit as $k \rightarrow 0, a_{k}$ approaches an even function with maximum or minimum at the origin, and a limit approached as $x \rightarrow \pm \infty$. In addition, corresponding to $k=0$, there are constant bifurcating solutions. These are obtained by dropping the diffusion term $D u_{x x}$ in (4.1), and applying simple bifurcation techniques.

We now come to the question of stability. It is as yet unclear which of the bifurcating periodic solutions mentioned are stable. The proper formulation of the linearized stability criterion for periodic stationary solutions was given by Kopell and Howard (1973) (in the context of wave trains, but certainly applicable here), but the linearized criterion is not easy to check. In the case of the bifurcating constant solutions alluded to last in the above paragraph, standard bifurcation theory tells us which are stable to perturbations independent of $x$. Possibly in the case $k_{0}=0$, these are stable to small bounded $x$-dependent perturbations as well, and they are the only ones so stable. This is an attractive conjecture to make, since the constant solutions are singled out from the others as being special, and because such a result holds in the case of a scalar equation. Yet besides these, there appear to be no good a priori reasons to think that they are the stable ones. In the case $k_{0} \neq 0$, certain special periodic solutions also appear to be singled out: those with maximum amplitude as $k$ is varied, for fixed $\lambda$. The same remark holds here as well.

It should be noted that the whole question of the appearance of small 
amplitude solutions of (4.1) is analogous to the Bénard problem and other bifurcation problems in fluid dynamics, posed in an infinite domain. The latter problems, allowing refined experimental investigation, have in addition been the subject of a considerable body of theoretical knowledge (for a good overview emphasizing stability questions, see Joseph (1976)). Most of the techniques used in studying infinite-domain bifurcation problems in fluid dynamics can be applied to reaction-diffusion equations as well, and we can therefore expect that at least some heuristic stability results can be obtained for the bifurcating periodic solutions we have found.

For the same reason, it is to be expected that two- or three-dimensional bifurcation patterns can be constructed. This is a subject for further investigation. Particularly suggestive here are the group theoretical techniques found in Sattinger (1978b, c) and elsewhere.

(b) Larger amplitude solutions: peaks. Systems (4.1) admitting larger amplitude stationary solutions are easy to devise. Linear systems with constant coefficients, for example, admit exponential solutions, and it is a simple matter to arrange the coefficients so that solutions sinusoidal in $x$ and independent of $t$ exist. Alternatively, one can take $m=1$ and produce periodic and peaked solutions of the resulting nonlinear scalar equations, as in §3. Unfortunately, the latter type of stationary solution is always unstable, as we have shown. The former situation is that treated in $\$ 4(a)$, with $g$ (in (4.2)) identically zero. The solution in question is $u=\Phi e^{i k_{0} x}$, where $\Phi$ is the nullvector of $H\left(k_{0}^{2}, \lambda_{0}\right)$. (We actually get two linearly independent solutions by taking the real and imaginary parts.) a small deviation of $\lambda$ away from $\lambda_{0}$ in one direction or another will usually replace the stationary solutions with solutions which grow exponentially in time, so the former will be a structurally unstable phenomenon. Structurally stable patterns exist only for nonlinear systems; and for this reason we omit any further consideration of linear ones.

So we concentrate our search within the class of truly nonlinear systems of more than one equation. First, we mention that reaction-diffusion systems have been proposed by various researchers to model, on a primitive level, pattern formation in biological contexts. These include the famous "Brusselator" equations (with diffusion added) and other models of the group in Brussels. Their extensive work has been reported in Herschkowitz-Kaufmann and Nicolis (1972), Nicolis (1974), and elsewhere. Also in this category are the activator-inhibitor equations of the group in Tübingen (Gierer and Meinhardt (1972), (1974), and other papers). In both cases, computer simulations have produced apparently stable large-amplitude stationary structures. These models have involved systems of two equations.

In the case of the equations developed by Gierer and Meinhardt, a heuristic argument was given to indicate why stable peaks and patterns may be expected. They characterized their two reacting and diffusing substances as a short-range activator and a long-range inhibitor. We may interpret lengths of ranges as being measured by the size of the respective diffusion coefficients. Recently Keener (1978) has provided an asymptotic analysis of these equations on a bounded spatial interval, based on the largeness of one of the diffusion coefficients. Stability was not considered. 
As far as rigorous stability proofs for large-amplitude stationary solutions are concerned, only the following example is known. Consider the problem of finding a reaction-diffusion system which has a given function of $x$ as stable stationary solution. This inverse approach has been successful to an extent, as shown by the following result (Fife (1977b)).

THEOREM. Let $\phi: \mathbf{R} \rightarrow \mathbf{R}$ be a bounded nonconstant function such that $\phi^{\prime \prime}=F(\phi)$ for some $C^{2}$ function $F$. (Then from symmetry considerations, $\phi$ will be periodic, peaked-with a single maximum or minimum and limit as $|x| \rightarrow$ $\infty$-or monotone.) Let $\psi$ satisfy $\phi=c_{1} \psi+c_{2}, c_{1}>0$. Let $k>1$. Then there exist functions $f(u, v), g(u, v)$ such that $(\phi, \psi)$ is a marginally stable (lin. crit.) stationary solution of

$$
u_{t}=u_{x x}+f(u, v), \quad v_{t}=k v_{x x}+g(u, v) .
$$

In the cases when $\phi$ is peaked or monotone and $F^{\prime}\left(\phi_{i}\right)>0$ for any limit $\phi_{i}$ of $\phi$ as $x \rightarrow \pm \infty$, then $(\phi, \psi)$ is strictly stable in the sense of $\$ 2$.

The functions $f$ and $g$ can be given explicitly; and $u$ and $v$ can be interpreted as an "activator" and "inhibitor", in the sense that

$$
f_{u}(\phi, \psi)>0, \quad g_{u}(\phi, \psi)>0,
$$

with the opposite inequalities holding for $f_{v}$ and $g_{v}$. Furthermore the fact that $k>1$ (which is an essential hypothesis) means that the inhibitor $v$ has "larger range" than that of the activator $u$. The activator-inhibitor properties of the Gierer-Meinhardt system, in fact, were the guiding principle behind the above construction.

In the proof of the above result, a stability criterion of Sattinger (1976a) is used. Briefly paraphrased, the criterion is that the operator obtained by linearizing the right-hand side of (4.1) about the given solution have spectrum to the left of, and bounded away from, the imaginary axis, except for a simple eigenvalue at the origin (which must always exist, because of the autonomous nature of the system and the infinite domain). Certain other reasonable conditions on the spectrum are also required. The stability criterion applies to traveling waves in general. But to my knowledge, this is the first application of Sattinger's theorem to a system of more than one equation. The required properties of the linearized operator are established by using known spectral properties of the second order operator $L w=w^{\prime \prime}-F^{\prime}(\phi) w$.

(c) Larger amplitude solutions: asymptotic methods. The methods of asymptotic analysis open a rich field of opportunities for the construction, on a formal basis, of stationary solutions of (4.1) (the same is true of traveling wave solutions, as we shall see). These methods are sometimes applicable when small or large parameters appear in the matrix $D$ or function $f$. Their purpose is to reduce the analysis of a given system (4.1) to the analysis of a lower order one, possibly with the additional complication that solutions of the lower order system may have to be patched in some manner. Such a reduction, which exploits the smallness of the parameter, is generally only formal and approximate. However in some cases (Fife (1976a), for example) it can be justified, in the sense that an exact solution which is close (distance related to the small parameter) to the formal solution can be proved to exist.

The procedure can best be seen by considering a pair of equations, one of 
them with small diffusion coefficient $\varepsilon^{2}$. Looking for stationary solutions, we drop the time derivative:

$$
\begin{array}{r}
\varepsilon^{2} \Delta u+f(u, v)=0, \\
\Delta v+g(u, v)=0 .
\end{array}
$$

As a first approximation, we look for a pair $\left(u_{0}(x), v_{0}(x)\right)$ satisfying, for all $x$, this pair of equations with $\varepsilon=0$ :

$$
\begin{aligned}
f\left(u_{0}, v_{0}\right) & =0 \\
\Delta v_{0}+g\left(u_{0}, v_{0}\right) & =0 .
\end{aligned}
$$

If the first equation may be solved for $u_{0}$, say $u_{0}=h\left(v_{0}\right)$, then this latter problem reduces to a single equation

$$
\Delta v_{0}+g\left(h\left(v_{0}\right), v_{0}\right)=0
$$

and we can seek patterned solutions of the latter. But they are unstable within the confines of that single equation, as we have seen in $\$ 3$.

A more promising situation occurs when (4.5a) has more than one solution, $u_{0}=h_{i}\left(v_{0}\right), i=1,2$. Then the possibility exists of using the function $h_{1}$ (say) to obtain a first approximation for some values of $x$, and $h_{2}$ for others. Thus, for two subsets $\mathscr{D}_{1}$ and $\mathscr{D}_{2}$ of $\mathbf{R}^{n}$,

$$
\Delta v_{0}+g\left(h_{i}\left(v_{0}\right), v_{0}\right)=0 \text { in } \mathscr{D}_{i} \text {. }
$$

This idea is explained for the case of one space variable in Fife (1977a). There, $\mathscr{D}_{1}$ and $\mathscr{D}_{2}$ are infinite collections of intervals alternating one with another. The lengths of the intervals may not be chosen arbitrarily, as we shall see. Since $u_{0}=h_{i}\left(v_{0}\right)$ and $v_{0}$ is continuous, this first approximation shows $u_{0}$ to be discontinuous in passing from one interval to adjacent ones.

The actual solution is not discontinuous, of course, but rather exhibits a layer effect (an abrupt transition in an interval of width $O(\varepsilon)$ ) at these transition points. Further information can be obtained by matched asymptotic analysis. This technique proceeds by recognizing that the solution has two natural length scales: one is the original length scale used in setting up the problem, and the other measures length in units smaller, by a factor $\varepsilon$, than the original units. The solution $u$ in the transition layers is found by using the smaller length scale. This is done by defining $\xi=\left(x-x_{0}\right) / \varepsilon$, where $x_{0}$ is the location of any transition point from an interval of $\mathscr{D}_{1}$ to one of $\mathscr{D}_{2}$, and rewriting (4.5a) in terms of $\xi$ :

$$
u_{\xi \xi}+f(u, \hat{v})=0 .
$$

Here $\hat{v}$ is the value of $v$ at the transition point. Now $v_{0}(x)$ is a solution of (4.7) and is therefore continuous with its first derivative, even though the nonlinear term in the equation is discontinuous. Therefore $\hat{v}$ is well defined.

In standard parlance, "matching" conditions must be imposed on the solution of (4.8). They constitute boundary conditions at $\xi= \pm \infty$, and are (if the transition is from $\mathscr{D}_{1}$ on the left to $\mathscr{D}_{2}$ on the right, say)

$$
\lim _{\xi \rightarrow-\infty} u(\xi)=h_{1}(\hat{v}), \quad \lim _{\xi \rightarrow \infty} u(\xi)=h_{2}(\hat{v}) .
$$


It turns out that (4.8), (4.9) has a solution if and only if the following condition is satisfied:

$$
\int_{h_{1}(\hat{v})}^{h_{2}(\hat{v})} f(u, \hat{v}) d u=0
$$

This condition will, in the simplest cases, be satisfied for only one value of $\hat{v}$, and will therefore serve to determine the value of $v_{0}$ at the transition points.

The problem for the first approximation $v_{0}$ has now been reduced to the following: Solve (4.7), subject to the condition $v_{0}=\hat{v}$ on the boundaries between $\mathscr{D}_{1}$ and $\mathscr{D}_{2}$. Of course, the sets $\mathscr{D}_{i}$ are to be determined in the process.

An alternate formulation is possible, if the functions $G_{i}(v) \equiv g\left(h_{i}(v), v\right)$ are each of a single sign, the sign being opposite for $i=1$ and 2. Suppose, for example, that $G_{1}(v)<0<G_{2}(v)$ for all $v$. Then the maximum principle implies that any solution of $\Delta v_{0}+G_{1}\left(v_{0}\right)=0$ in $\mathscr{D}_{1}, v_{0}=\hat{v}$ on $\partial \mathscr{D}_{1}$, must satisfy $v_{0}(x)<\hat{v}$ in the interior of $\mathscr{D}_{1}$. Similarly $v_{0}>\hat{v}$ in $\mathscr{D}_{2}$. Thus our problem becomes

$$
\Delta v_{0}+G\left(v_{0}\right)=0 \text { in } \mathbf{R}^{n}
$$

where

$$
G(v) \equiv \begin{cases}G_{1}(v), & v<\hat{v} \\ G_{2}(v), & v>\hat{v}\end{cases}
$$

In Fife (1977a) conditions were given under which it is reasonable to expect the existence of a one-parameter family of periodic solutions of this problem, in one space dimension. Moreover, further conditions were given under which, according to a heuristic argument, the resulting patterns will be stable with reference to the original system (4.1).

It seems reasonable to expect that doubly periodic solutions exist when $n=2$. A special example is the case when $\hat{v}=0, G_{1}(v) \equiv 1, G_{2}(v) \equiv-1$. Let $S$ be any square in the $\left(x_{1}, x_{2}\right)$ plane. Solve the Dirichlet problem $\Delta v_{0}=-1$ in $S, v_{0}=0$ on $\partial S$. Then continue $v_{0}$ as an odd function with respect to each of the four sides of $S$. In adjacent squares, the continued function will satisfy $\Delta v_{0}=1=-G_{2}\left(v_{0}\right)$. Continuing in this way, we obtain a checkerboard pattern with $u_{0}$ alternately $h_{1}\left(v_{0}\right)$ and $h_{2}\left(v_{0}\right)$ on the squares (see Figure 3$)$. Such a solution is possible for any size of square.

Now let us perturb the problem by having $G_{1}(v) \equiv 1+\varepsilon$, with $G_{2}$ remaining -1 , and conjecture about the fate of the original pattern. The symmetry is destroyed, and such a rectilinear checkerboard pattern is no longer possible. But it is plausible that patterns with curvilinear sides appear, as shown by the dotted lines in Figure 3. The effect of the curves will be to reduce the size of those "squares" wherein $\Delta v_{0}=-G_{1}$, relative to the others. Such a reduction is necessary, for the following reason. The pattern is constructed by periodically repeating the basic configuration of four adjacent "squares." Call this block of four the domain $T$. By periodicity, $0=\int_{\partial T}\left(\partial v_{0} / \partial \nu\right) d s$, where $\partial v_{0} / \partial \nu$ is the outward normal derivative. But this line integral is equal to

$$
\iint_{T} \Delta v_{0} d x=-\iint_{T} G\left(v_{0}\right) d x=-\iint_{T_{1}}(1+\varepsilon) d x+\iint_{T_{2}} d x
$$


where $T_{i}$ is the diagonal pair of "squares" in the block where $G=G_{i}$. Thus

$$
(1+\varepsilon) \iint_{T_{1}}=\iint_{T_{2}}
$$

which means that the "squares" in $T_{1}$ must be smaller in area than those of $T_{2}$, by a factor $1 /(1+\varepsilon)$.

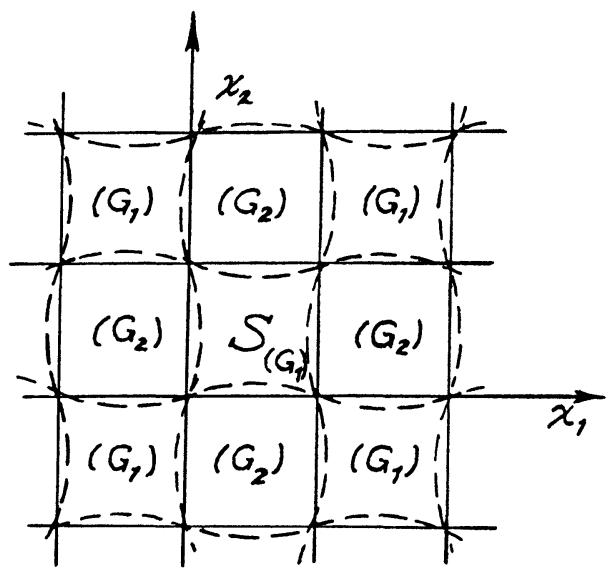

FIGURE 3

5. Plane wave trains. Plane wave trains are defined as solutions of (4.1) of the form $u(x, t)=\tilde{u}(x \cdot \nu-c t)$, where $\tilde{u}$ is a periodic function of the single variable $x \cdot \nu-c t, c$ is the velocity, and $\nu$ is a unit vector (the direction of propagation). It clearly suffices to pose the problem in one space dimension (distances measured in the direction of $\nu$ ), and we shall do so. Letting $k$ equal the wave number ( $k=2 \pi /$ wave length) and setting $\bar{\omega}=-k c$, we have that wave trains are $2 \pi$-periodic functions of the variable $z=k x+\bar{\omega} t$. Here $\bar{\omega}$ is the frequency of oscillation at any fixed value of $x$. Setting $u=U(z)$, we see that (4.1) becomes

$$
k^{2} D U^{\prime \prime}-\bar{\omega} U^{\prime}+f(U)=0 .
$$

The frequency $\bar{\omega}$ is conceptually distinct from $\omega=\operatorname{Im} \mu_{1}$ defined previously in $\$ 4(a)$.

Chemical concentration wave trains have been observed (Zaikin and Zhabotinsky (1970)) in solutions supporting the Belousov reaction, one of the better known "exotic" chemical reactions capable of producing oscillations and other effects. The observed patterns were circular rather than planar, but it is clear that plane wave trains approximate the observed ones at large distances from the center.

The mathematical analysis of plane wave trains is due principally to Kopell and Howard (1973). (Gmitro and Scriven (1966) had much earlier observed that a linearized analysis of reaction-diffusion equations suggests the possibility of small amplitude wave trains.) Kopell and Howard proved the existence of families of small amplitude ones under certain circumstances, and also the existence of long wave-length trains under the assumption that the kinetic equations 


$$
d u / d t=f(u)
$$

have a stable limit cycle. Ortoleva and Ross (1974) also studied these two types of wave trains in their treatment of chemical wave phenomena.

In both of these references, the authors point out that the small amplitude wave trains constructed are necessarily unstable. The reason for this can be seen by reference to the framework in our $\$ 4$. Consider the linear equation (4.3) and the eigenvalue $\mu_{1}\left(k^{2}, \lambda\right)$ defined therefrom. The assumptions made by the above authors differ from our Assumption $A$ in that (ii) is replaced by the condition that $\partial \sigma\left(k_{0}^{2}, \lambda_{0}\right) / \partial k^{2} \neq 0$, so that there are values of $k$ near $k_{0}$ for which $\sigma>0$. This implies that every bounded solution of the linearized version (4.3) is unstable, because an exponentially growing solution with an unstable wave number $k$ can always be added to it. Likewise, every small bounded solution of the nonlinear equation (4.1), (4.2) will be unstable for $\lambda$ near $\lambda_{0}$, for by continuity, the spectrum of the operator linearized about it will also contain points in the unstable half-plane.

Nevertheless, it is undoubtedly true that stable small amplitude wave trains do sometimes exist, and that they can be obtained as bifurcating solutions when Assumption A holds, with $\omega_{0} \neq 0$. We explore this in $\$ 5(a)$.

$\$ 5$ (b) will involve the question of wave trains obtained from a limit cycle; we follow Ortoleva and Ross in calling them "wave extensions from homogeneous oscillations." In $\$ \S 5(\mathrm{c})$, (d), we point out that wave trains have been shown to exist for special equations: those of nerve impulse propagation and those of predator-prey dynamics.

(a) Small amplitude wave trains. The function $f$ is taken to depend on a real parameter $\lambda$ as in (4.2). We make Assumption A, and also suppose $\omega_{0} \neq 0$.

The problem is to find a family of $2 \pi$-periodic solutions of (5.1), allowing the possibility that in this family, relations may be necessary between the parameters $\bar{\omega}, \lambda$, and the "amplitude" $\varepsilon$. Actually, we shall also allow $k$ to vary. In the treatment given by Kopell and Howard (1973), $\lambda$ did not enter into the problem in the first place, so in our context would be held fixed, and the relations were such that $k$ and $\bar{\omega}$ depended on $\varepsilon$. Then specific conditions on $D$ and $A$ were given under which a bifurcation of wave trains takes place. In our treatment, $\lambda$ and $\bar{\omega}$ will depend on the two independent parameters $\varepsilon$ and $k$ ( $\varepsilon$ and $k-k_{0}$ restricted to be small enough).

If the matrix $k_{0}^{2} D$ is nonsingular, then (5.1) may be written as a system of $2 m$ first order equations, and the existence of the required solutions proven as in Kopell and Howard (1973) through use of the Hopf bifurcation theorem. It is practically as easy, however, to show that at least one proof of the Hopf result goes through when suitably modified to handle (5.1) directly, rather than a system of first order equations. We shall sketch how this proceeds, using an argument along the lines of that of Crandall and Rabinowitz (1978).

As stated, we operate under Assumption A with $\omega_{0} \neq 0$. We take $\lambda_{0}=0$, $k_{0} \neq 0$. Thus, the matrix $H_{0}=-k_{0}^{2} D+A$ has algebraically simple eigenvalues $\pm i \omega_{0}$, and $H\left(k^{2}, 0\right)=-k^{2} D+A$ has no other purely imaginary eigenvalues for any value of $k$. Let $\Phi$ be a suitably normalized eigenvector of $H_{0}$, associated with $i \omega_{0}$. In the space of real continuous $2 \pi$-periodic functions of $z$ (call it $C_{0}^{p}$ ), the operator 


$$
L U \equiv k_{0}^{2} D U^{\prime \prime}-\omega_{0} U^{\prime}+A U
$$

has a two-dimensional nullspace spanned by

$$
\phi_{1}=\frac{1}{2}\left(\Phi e^{i z}+\bar{\Phi} e^{-i z}\right)=\operatorname{Re} \Phi e^{i z}
$$

and

$$
\phi_{2}=\phi_{1}^{\prime} \text {. }
$$

It can also easily be shown (Crandall and Rabinowitz (1978)) that the adjoint operator $L^{*} U \equiv k_{0}^{2} D^{*} U^{\prime \prime}+\omega_{0} U^{\prime}+A^{*} U$ has nullvectors $\psi_{1}(z)$ and $\psi_{2}(z)=\psi_{1}^{\prime}(z)$ with the properties

$$
\left(\phi_{i}, \psi_{j}\right)=\delta_{i j},
$$

where the expression on the left is the scalar product in $\ell_{2}(0,2 \pi)$.

We look for a $2 \pi$-periodic solution of (5.1) in the form

$$
U=\varepsilon\left(\phi_{1}+w\right),
$$

where $w$ is orthogonal to $\operatorname{span}\left\{\phi_{1}, \phi_{2}\right\}=$ nullspace of $L$. This is analogous to the procedure outlined in $\$ 4(\mathrm{a})$. The results are also analogous to a certain extent, but are actually simpler because in the present case there is an extra adjustable parameter, in the form of the frequency $\bar{\omega}$ (of course, we pay for this because the presence of the first derivative term in the equation destroys a reflection symmetry the equation originally had).

We substitute (5.4) into (5.1), and at the same time set $k^{2}=k_{0}^{2}+q$, $\bar{\omega}=\omega_{0}+\zeta$, and $h(u, \varepsilon)=g(\varepsilon u) / \varepsilon^{2}$, where $g$ is from (4.2). Thus

$$
L w-\zeta\left(\phi_{1}^{\prime}+w^{\prime}\right)+\lambda B\left(\phi_{1}+w\right)+q D\left(\phi_{1}^{\prime \prime}+w^{\prime \prime}\right)+\varepsilon h\left(\phi_{1}+w, \varepsilon\right)=0 \text {. }
$$

Let $P_{i}(i=1,2)$ denote the orthogonal (in the sense of $\left.L_{2}(0,2 \pi)\right)$ projection onto $\operatorname{span}\left\{\psi_{i}\right\}$, and $Q=I-P_{1}-P_{2}$. Then (5.5) may be decomposed into three components, obtained by applying, in turn, the three projections $Q, P_{1}$, and $P_{2}$. The operator $L$, being Fredholm, satisfies $P_{i} L=0, Q L=L$. We therefore obtain (setting $\phi=\phi_{1}$ )

$$
\begin{aligned}
L w+Q\left\{-\zeta\left(\phi^{\prime}+w^{\prime}\right)\right. & +\lambda B(\phi+w) \\
& \left.+q D\left(\phi^{\prime \prime}+w^{\prime \prime}\right)+\varepsilon h(\phi+w, \varepsilon)\right\}=0, \\
-\zeta P_{1} \phi^{\prime}+\lambda P_{1} B \phi= & P_{1}\left\{\zeta w^{\prime}-\lambda B w-q D\left(\phi^{\prime \prime}+w^{\prime \prime}\right)-\varepsilon h\right\} \\
& -\zeta P_{2} \phi^{\prime}+\lambda P_{2} B \phi=P_{2}\{\ldots\} .
\end{aligned}
$$

Let us be a little more precise about the domains of the above operators. We let $C_{j}^{p}$ be the space of functions on $\mathbf{R}$, continuous with their derivatives to order $j$, and $2 \pi$-periodic. The usual $C_{j}$ norm is used. Let $X$ denote the $\ell_{2}(0,2 \pi)$-orthocomplement of span $\left\{\phi_{1}, \phi_{2}\right\}$ in $C_{2}^{p}$, and $Y=Q C_{0}^{p}$. We interpret $L$ as an operator from $X$ to $Y$. If $D$ is nonsingular, which we assume, then it is known that $L$ has a bounded inverse.

Now (5.6a) may be solved for $w$ as a function of the four small parameters $\zeta, \lambda, q$ and $\varepsilon$. This can be done by the implicit function theorem, using the invertibility of $L$. 
With $w$ so determined, we solve (5.6b), (5.6c) for $\zeta$ and $\lambda$ as functions of $\varepsilon$ and $q$, again by the implicit function theorem. This is a triviality, once one realizes that (5.3) implies $P_{1} \phi^{\prime}=0, P_{2} \phi^{\prime} \neq 0$, and that condition (iii) of Assumption A implies $P_{1} B \phi \neq 0$ (Fife (1977a)).

With this, the following theorem is obtained.

THEOREM. Under Assumption A and the additional assumption that $\omega_{0} \neq 0$, $k_{0} \neq 0$, and $D$ is nonsingular, there exists a two-parameter family $(U(z ; \varepsilon, k)$, $\lambda(\varepsilon, k), \bar{\omega}(\varepsilon, k))$ of solutions of $(5.1)$, defined and continuous in $(\varepsilon, k)$ for small $\varepsilon$ and $\left|k-k_{0}\right|$, such that

(i) $U$ is $2 \pi$-periodic in $z$,

(ii) $\operatorname{Max}_{z}|U|=\varepsilon$,

(iii) $\lambda\left(0, k_{0}\right)=\lambda_{0}, \bar{\omega}\left(0, k_{0}\right)=\omega_{0}$.

If we now allow that $k_{0}=0$ and/or $D$ be singular, then a formal solution can still be found in powers of $\varepsilon$ and $q$, though its existence has not yet been proved.

The stability of the wave trains so constructed has yet to be investigated in detail, but there appears to be little doubt that some of them will be stable. As mentioned in the preceding section, the correct approach for a linearized stability analysis of them was given in Kopell and Howard (1973).

(b) Wave extensions from homogeneous oscillations. We now suppose that the kinetic equations

$$
d u / d t=f(u)
$$

have a limit cycle, $u=u_{0}(t)$, with some minimal period $T=2 \pi / \omega_{0}$. We assume it to be stable, in the sense that the Floquet matrix of the linearized system

$$
d y / d t=f^{\prime}\left(u_{0}(t)\right) y
$$

has 1 as a simple eigenvalue, and all other eigenvalues $\lambda$ have $|\lambda|<1$.

Again, we seek $2 \pi$-periodic solutions of (5.1). There turns out to be a family $\left(U_{k}, \bar{\omega}_{k}\right)$ of such solutions, parameterized by the wave number $k$, and defined for small $k$ (hence large wave length). As $k \rightarrow 0, \bar{\omega}_{k} \rightarrow \omega_{0}$ and $U_{k}$ approaches the originally given periodic solution, $U_{0}\left(\omega_{0} t\right) \equiv u_{0}(t)$. At the same time, their velocity $c=\bar{\omega} / k \rightarrow \infty$.

The existence of these rapid long wave-length wave trains has been established by a variety of methods, two of them found in Kopell and Howard (1973) (K-H). Ortoleva and Ross (1974) (O-R) indicate that the question can be formulated as a singular perturbation problem for which the hypotheses of an existence theorem of Wasow (1965) can be verified. The existence then follows from that theorem. These authors also give an asymptotic method for the construction of approximations, based on the smallness of $k$.

As expected, a simple stability criterion has not been given, except in the special case of $\lambda-\omega$ systems (see below). The analysis of this case tells us that the wave extensions of stable limit cycles are often, but not always, stable for all small values of $k$.

Kopell and Howard (1973) introduced a special class of systems of reaction-diffusion equations, called " $\lambda-\omega$ systems." They have proved very 
instructive, because on the one hand, they exhibit much of the rich variety of phenomena one expects from more general systems with $m>1$, and yet on the other hand, important solutions of special types (wave trains, target patterns, homogeneous oscillations) admit a far more complete analysis than is generally the case in systems with $m>1$.

This type of system has $m=2$ in (2.1), and

$$
f(u)=\left[\begin{array}{cc}
\lambda(r), & -\omega(r) \\
\omega(r), & \lambda(r)
\end{array}\right]\left[\begin{array}{l}
u_{1} \\
u_{2}
\end{array}\right],
$$

where $r^{2}=|u|^{2}$, and $\lambda$ and $\omega$ are any given functions of $r$.

As regards wave train solutions, global families (not just families with small amplitude or large wave length) can be exhibited (K-H, O-R), and their stability analyzed (K-H).

There may exist wave trains with the properties both of those treated in our $\$ 5(a)$, and of those in 5(b); that is to say, with small amplitude and long wave length. This situation is simply that in 5(a) with the exception that $k_{0}=0$. Now one looks for solutions depending on two small parameters, namely the wave number and the amplitude. The parameter $\lambda$ must also be sought as a function of these two parameters. This, of course, is the case when the kinetic equations (5.7) undergo a Hopf bifurcation at $\lambda=\lambda_{0}$ : whereas a trivial solution $u=0$ exists for all $\lambda$, other periodic solutions of (5.7) appear when $\lambda>\lambda_{0}$. A formal analysis shows rapid wave trains to exist as well.

(c) Wave trains in excitable media. Wave train solutions of the HodgkinHuxley and FitzHugh-Nagumo systems from neurophysics have been shown to exist. This work will be discussed in $\$ 7$, along with pulse solutions of the same systems.

(d) Wave trains for predator-prey equations. Consider equations of the type

$$
u_{t}=a u_{x x}+u f(u, v), \quad v_{t}=b v_{x x}+v g(u, v) .
$$

These can be interpreted as classical predator-prey equations ( $u$ being the prey density) with spatial diffusion added, if $f_{v}<0$ and $g_{u}>0$. Conley and Smoller (in preparation) have proven the existence of wave train solutions when certain conditions are satisfied. For example, their theorem holds when

(1) the zero sets of $f$ and $g$ are as shown in Figure 4 so that all orbits of the corresponding kinetic equations tend to a rest point on an axis (at least one species always goes extinct), and

(2) $a \gg b$.

In this peculiar way, the presence of diffusion can prevent extinction.

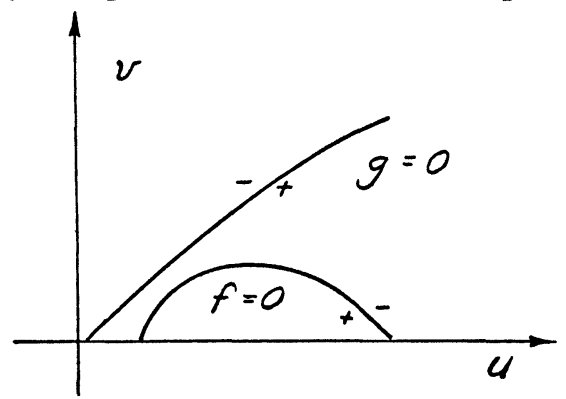

FIGURE 4 
6. Plane wave fronts. As with wave trains, fronts are solutions which depend on the single combination of variables $z=x \cdot \nu-c t: u=U(z)$. But rather than being a periodic function of $z$, a front approaches distinct limits as $z \rightarrow \pm \infty$. As we have seen in $\$ 3$, fronts and combinations of them account for all the known nontrivial stable asymptotic states for the scalar nonlinear diffusion equation. They undoubtedly form an important class of states for systems as well, but at present not many results are available.

Of course, wave fronts must connect two rest states of the kinetic equations (5.2), in that $f(U( \pm \infty))=0$; this can be seen by passing to the limit as $z \rightarrow \pm \infty$ in (5.1). Moreover, it is easy to see that for a wave front to be $C_{0}$-stable for (4.1), it is necessary for the two rest states $U( \pm \infty)$ to be stable for (5.2). With these two necessary conditions in mind, one can now formulate the converse problem:

Problem. Given two stable rest states $U_{-}$and $U_{+}$of (5.2), find conditions under which there exists a wave front $U(z)$ with $U( \pm \infty)=U_{ \pm}$.

To my knowledge, the following are the only (partial) answers to this problem, for systems of more than one equation.

(a) Gradient systems. If the function $f$ can be written as a gradient with respect to $u$,

$$
f(u)=\nabla h(u)
$$

for some scalar function $h$, or more generally if $(f(u), \nabla h(u))>0$ for $\nabla h(u) \neq 0$, and $u_{0}$ and $u_{1}$ are local maxima of $h$, then under nonrestrictive hypotheses, Conley (1977, IV.3.2) has shown that there does exist a wave front connecting $u_{0}$ to $u_{1}$. For simplicity, we state his result for the case when $D=d_{0} I$ ( $d_{0}$ a scalar), though it is readily generalizable to other cases.

THEOREM. Assume the above, where $h: \mathbf{R}^{n} \rightarrow \mathbf{R}^{1}$ is smooth and satisfies

(1) $h(u) \rightarrow-\infty$ as $|u| \rightarrow \infty$, and there exists a (negative) constant $C_{0}$ such that $\{u \mid h(u) \geqslant C\}$ is convex whenever $C<C_{0}$.

We normalize so that $h$ assumes its absolute maximum at $0=u_{0}$, and the maximum is 0.

(2) $2 h(u)+(u, \nabla h(u))<0$ for $u \neq 0$.

(3) $h$ has a local maximum at $u_{1}$, and for some constant $C_{1}<h\left(u_{1}\right)$, the set $\left\{u \mid h(u) \geqslant C_{1}\right\}$ contains only two critical points, namely 0 and $u_{1}$.

Then (4.1) has a solution of the form $u=U(x-c t)$ for some $c>0$, with $U(-\infty)=0, U(\infty)=u_{1}$.

Conley's methods, crudely stated, are based on algebraic properties of flows which remain invariant under certain deformations. These methods have had applications in many fields. See Conley (1978) for the most complete account.

(b) Small amplitude fronts. Bifurcation of wave fronts can occur in a setting very similar to that of bifurcation of stationary periodic solutions in 4(a), or bifurcation of wave trains in 5(a). We again assume a parameter dependence of the form (4.2), and again subscribe to Assumption A. We suppose that $\omega_{0}=k_{0}=0$. This means that the matrix $A$ has 0 as an algebraically simple eigenvalue, with corresponding eigenvector $\Phi$. Also for simplicity, assume that $\lambda_{0}=0$. This is the setting in which periodic long wave-length stationary 
solutions may exist, as shown in $\$ 4(\mathrm{a})$. Now we show that slow wave fronts can also exist, provided the function $g$ in (4.2) is cubic, rather than quadratic, to lowest order in $u$ (or, satisfies (3) below, which is more general).

Our specific assumptions, in addition to Assumption A, are as follows. Here we denote by $\Psi$ the unit real nullvector of $A^{*}$, the transpose of $A$.

(1) $\alpha \equiv(D \Phi, \Psi) \neq 0$,

(2) $\beta \equiv(B \Phi, \Psi) \neq 0$,

(3) $\lim _{\varepsilon \rightarrow 0} \varepsilon^{-2}(g(\varepsilon \Phi), \Psi)=0$,

(4) $\eta=\lim _{\varepsilon \rightarrow 0} \varepsilon^{-3}(g(\varepsilon \Phi), \Psi) \neq 0$,

(5) $\alpha \beta>0$ and $\beta \eta<0$.

The slow fronts can be constructed as functions of the variable $\zeta=\varepsilon(x-$ - $\left.c_{\mathrm{e}} t\right)$, and take the form

$$
u_{e}(x, t)=\varepsilon\left(a_{e}(\zeta) \Phi+\varepsilon^{2} w_{e}(\zeta)\right),
$$

here $a_{\mathrm{e}}$ is a scalar function satisfying

$$
\lim _{\zeta \rightarrow \infty} a_{e}(\zeta)=1
$$

(this normalization condition really serves to define $\varepsilon$ ), and $\left(\Phi, w_{\varepsilon}(\xi)\right)=0$ for each $\zeta$. Of course, $\varepsilon$ is a small parameter and the quantities $a_{e}$, $w_{e}$, and $c_{e}$ can be formally expanded in powers of $\varepsilon$. At present lacking an existence proof tor the solution (one could probably be provided), we simply describe the prccess of obtaining the formal expansion.

We substitute (6.1) into (4.1), using (4.2) and at the same time setting $\lambda=\varepsilon^{2} \gamma_{\varepsilon}$ The result is the following equation for $w, a$, and $\gamma$ :

$$
\begin{aligned}
A w+\{ & D\left(a \Phi+\varepsilon^{2} w\right)^{\prime \prime}+\gamma B\left(a \Phi+\varepsilon^{2} w\right) \\
& \left.+h\left(a \phi+\varepsilon^{2} w, \varepsilon\right)+\varepsilon c a^{\prime} \Phi+\varepsilon^{3} c w^{\prime}\right\}=0,
\end{aligned}
$$

where $h(v, \varepsilon) \equiv \varepsilon^{-3} g(\varepsilon v)$.

For the existence of a solution, the quantity in braces must be orthogonal to $\Psi$ at each point $\xi$. Considering the above definitions of $\alpha, \beta, \eta$, we obtain the equation

$$
\alpha a^{\prime \prime}+\gamma \beta a+\eta a^{3}+\varepsilon\left[c a^{\prime}(\Phi, \Psi)+\left(\partial_{e} h(a \Phi, 0), \Psi\right)+O(\varepsilon)\right]=0 .
$$

setting $\varepsilon=0$ we obtain an equation satisfied by our lowest order approximations $a_{0}, \gamma_{0}$ to $a$ and $\gamma$ :

$$
a_{0}^{\prime \prime}+\gamma_{0} \frac{\beta}{\alpha} a_{0}+\frac{\eta}{\alpha} a_{0}^{3}=0 .
$$

If $\gamma_{0}>0$, the function

$$
F\left(a_{0}\right)=\frac{\gamma_{0} \beta}{\alpha} a_{0}+\frac{\eta}{\alpha} a_{0}^{3}
$$

has zeros at $a_{0}=0, \pm \sqrt{-\gamma_{0} \beta / \eta}$. Moreover by the discussion in $\$ 3$, there exists a solution of (6.5) with $a_{0}( \pm \infty)= \pm \sqrt{-\gamma_{0} \beta / \eta}$. (This can be considered a wave front solution of a scalar nonlinear diffusion equation (6.5) with zero velocity.) Because of our normalization condition (6.2), we must require 


$$
\gamma_{0}=-\eta / \beta>0,
$$

so that $a_{0}( \pm \infty)= \pm 1$.

In addition to (6.2), we also normalize $a_{\varepsilon}$ by translating the independent variable $\zeta$ so that

$$
a_{e}(0)=0 .
$$

This, applied to $a_{0}$, serves to determine the latter uniquely.

With the orthogonality condition satisfied to lowest order, the first approximation $w_{0}$ to $w$ may be obtained from (6.3) with $\varepsilon=0$. There exists a unique solution with $\left(w_{0}(\zeta), \Psi\right) \equiv 0$.

To obtain higher order approximations, we imagine that

$$
a_{\varepsilon}(\zeta)=a_{0}(\zeta)+\varepsilon a_{1}(\zeta)+\ldots, \quad w_{\varepsilon}(\zeta)=w_{0}(\zeta)+\varepsilon w_{1}(\zeta)+\ldots,
$$

with similar expansions for $c$ and $\gamma$.

Then from (6.4),

$$
\begin{aligned}
L a_{1} & \equiv \alpha a_{1}^{\prime \prime}+\gamma_{0} \beta a_{1}+3 \eta a_{0}(\zeta)^{2} a_{1} \\
& =-\gamma_{1} \beta a_{0}+c_{0} a_{0}^{\prime}(\Phi, \Psi)-\left(\partial_{\varepsilon} h\left(a_{0} \Phi, 0\right), \Psi\right),
\end{aligned}
$$

with $a_{1}(\infty)=0, a_{1}(0)=0$.

It turns out that $L$, as an operator in $C_{0}(-\infty, \infty)$, has a one-dimensional nullspace spanned by $a_{0}^{\prime}$, and that the equation $L a=p, p \in C_{0}$, has a solution if and only if

$$
\int_{-\infty}^{\infty} p(\zeta) a_{0}^{\prime}(\zeta) d \zeta=0
$$

We may deal with (6.8) by means of the following procedure.

(1) Choose $\gamma_{1}$ so that (6.8) is satisfied when $a_{1}^{\prime \prime}=a_{1}=a_{0}^{\prime}=0, a_{0}=1$. This will guarantee that any solutions we eventually obtain will satisfy $a_{1}(\infty)=0$.

(2) Choose $c_{0}$ so that the right-hand side of (6.8) is orthogonal to $a_{0}^{\prime}$, as described. This is clearly possible, since $(\Phi, \Psi) \neq 0$ (by the algebraic simplicity of the 0 eigenvalue of $A$ ). Then (6.8) will be solvable, and the solution will be unique up to an additive multiple of $a_{0}^{\prime}$.

(3) Choose the additive multiple so that $a_{1}(0)=0$.

Higher order terms are now obtained in any orderly fashion.

The peculiarity of this problem is that two quite distinct orthogonality conditions are used to effect successive reductions. The first, applied to (6.2), gives an ordinary differential equation (6.4). The second, applied to (6.4), gives us an equation for $c$.

The small-amplitude wave fronts so obtained serve to join uniform rest states of (4.1), i.e., zeros of $f(u, \lambda)=(A+\lambda B) u+g(u)$. These states can be obtained quite independently of the above, by standard bifurcation arguments. In fact there exists, besides the trivial solution $u=0$, a one-parameter family of rest states $\left(u_{e}, \lambda_{\varepsilon}\right)$ (solutions of $\left.f(u, \lambda)=0\right)$, defined for $|\varepsilon|<\varepsilon_{0}$. Moreover, for each $\lambda>\lambda_{0}$ with $\left(\lambda-\lambda_{0}\right)$ small enough, there exist two numbers $\varepsilon_{ \pm}(\lambda)$ with $\varepsilon_{-}(\lambda)<0<\varepsilon_{+}(\lambda)$ and $\lambda_{\varepsilon_{ \pm}}=\lambda$. The wave front indicated in the above theorem represents a transition from the rest state $u_{e_{-}}$to $u_{\varepsilon}$

For the wave front to be stable, we need to have these rest states stable 
solutions of the kinetic equations (5.2). Again, bifurcation theory tells us that will be true if the bifurcation is "supercritical," i.e., if $\lambda(\varepsilon)=\varepsilon^{2} \gamma(\varepsilon)>0$. But this is assured by (6.6), a consequence of our Assumption 5. This is only a necessary condition for the fronts' stability, and does not prove it. But it does lend a great deal of plausibility to the idea that they are stable.

(c) Systems with small parameters. A number of types of wave fronts can be formally constructed by techniques of matched asymptotics, which techniques also yield heuristic stability arguments. These possibilities have been explored by Ortoleva and Ross (1976), Fife (1976b, 1977c) and Feinn and Ortoleva (1977).

Moreover, in many cases, rigorous existence proofs for fronts like these have been obtained by Kurland (to appear) using topological methods due to Conley and others.

Here we shall be content to give an intuitive description of an example from one category of wave fronts which can be handled.

Consider the following system of two equations with small parameter $\varepsilon$ :

$$
\begin{aligned}
& u_{t}=\varepsilon u_{x x}+\varepsilon^{-1} f(u, v) \\
& v_{t}=v_{x x}+g(u, v) .
\end{aligned}
$$

We assume the system has stable rest states $(0,(0,0))$, and $(1,1)$. Thus $f$ and $g$ vanish at these two points. Let us assume the level lines $f=0$ and $g=0$ have the structure shown in Figure 5.

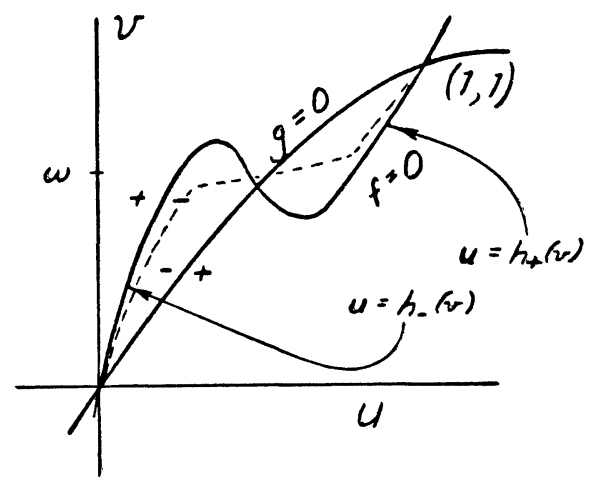

FIGURE 5

We look for solutions of (6.9) of the form $u=U(z, \varepsilon), v=V(z, \varepsilon)$, where $z=x-c t$, and where for each $\varepsilon,(U, V)$ passes from $(0,0)$ at $z=-\infty$ to $(1,1)$ at $z=+\infty$.

Taking a clue from the transition layer phenomena considered in $\$ 4(\mathrm{c})$, and using time-honored procedures from asymptotic analysis, we proceed under the ansatz that $U$ and $V$ are describable naturally in terms of two length scales, the smaller one appropriate in an abrupt "transition layer" or "shock" region, and the other one elsewhere.

First, we find an approximation away from the sharp layer. For this purpose, we substitute $u=U(z), v=V(z)$ into (6.9) to obtain

$$
\begin{array}{r}
\varepsilon^{2} U^{\prime \prime}+\varepsilon c U^{\prime}+f(U, V)=0, \\
V^{\prime \prime}+c V^{\prime}+g(U, V)=0,
\end{array}
$$


and then set $\varepsilon=0$ to obtain $f(U, V)=0$. This means that $(U, V)$ must lie approximately on the nullcurve $f=0$ in Figure 5. The middle, descending, part of that curve is a repeller for the kinetic equations $u_{t}=\varepsilon^{-1} f(u, v), v_{t}=$ $g(u, v)$, so we expect the solution cannot stay there in its region of slow variation. With the middle portion excluded, there remain the two ascending branches, expressible in the form $u=h_{ \pm}(v)$ (as shown). The transition layer, with smaller length scale, provides a means for the solution to pass from one branch $h_{-}$to the other, $h_{+}$(as shown by the dotted line in the figure). Such a transition is clearly necessary if the orbit is to connect the two rest states $(0,0)$ and $(1,1)$.

We normalize by letting $z=0$ be the location of the transition layer. Thus, outside this small layer and for $z<0$, the variables $U$ and $V$ are related approximately by $U=h_{-}(V)$. Away from the layer for $z>0$, however, approximately $U=h_{+}(V)$. As $z \rightarrow \pm \infty$, the rest points $(0,0)$ and $(1,1)$ are approached, as shown in Figure 5.

As in the situation analysed in $\$ 4(c), V$ will not change abruptly in the layer, so we may speak of the value of $V$ at the layer, say $V(0) \equiv \omega$. The equation for $V$, then, is obtained from (6.10b) and the above expression for $U$ :

$$
V^{\prime \prime}+c V^{\prime}+G_{\omega}(V)=0
$$

where

$$
G_{\omega}(V)= \begin{cases}g\left(h_{-}(V), V\right), & V<\omega \\ g\left(h_{+}(V), V\right), & V>\omega .\end{cases}
$$

This is the equation for a wave front solution of the scalar diffusion equation $v_{t}=v_{x x}+G_{\omega}(v)$, analogous to (3.1). By assumption $V=0$ and $V=1$ are both stable rest points for $V_{t}=G_{\omega}(V)$, so we are in the bistable case. Hence there is a unique solution $(V(z), c)$, depending on $\omega$ of course. Let $p$ denote the functional dependence of $c$ on $\omega: c=p(\omega)$.

We now examine the internal structure of the transition layer by using a smaller length scale. For a first approximation, we set $z=\varepsilon \zeta, V=\omega$, in (6.10a) to obtain

$$
U_{\zeta \zeta}+c U_{\zeta}+f(U, \omega)=0 .
$$

The procedure of matched asymptotics requires the boundary condition

$$
U \rightarrow h_{ \pm}(\omega) \text { as } \zeta \rightarrow \pm \infty \text {. }
$$

Again, this is a scalar wave front equation with unique velocity $c$, depending on $\omega: c=q(\omega)$.

For (6.11) and (6.12) to have solutions satisfying the required boundary conditions, it is therefore necessary that $\omega$ be chosen so that $p(\omega)=q(\omega)$. In the situation shown in Figure 5 , it is reasonable to expect that $p$ is an increasing, and $q$ a decreasing, function of $\omega$, and that both functions assume values of both signs. If this is indeed the case, then there will be a unique value of $\omega$ so determined. This completes the lowest order analysis of this type of wave front.

A heuristic argument can be given affirming the stability of this and similar 
types of fronts constructed by asymptotic analysis (Fife 1976b).

(d) A model from population genetics. Wave fronts in nonlinear diffusion problems were first introduced by Fisher (1937) in connection with natural selection in a biological population. As model, he used a scalar equation ( $\$ 3)$. So it may be appropriate that we devote a brief discussion here to a rather special reaction-diffusion system which provides a (presumably more accurate) model for natural selection with spatial diffusion, and which formally reduces to the Fisher equation when the selection parameter $\varepsilon$ approaches 0 . Conley (unpublished) has shown the existence of a wave front solution of this model system in cases when the corresponding scalar equation is of the bistable category, so has a stable front.

First, we consider a diploid population distributed continuously in space and time, separated into three subpopulations according to a simple genetic classification. We focus attention on one gene locus on a pair of chromosomes, and suppose that the locus may be occupied either by allele $a$ or by allele $A$. There result three classes of individuals (genotypes), denoted by $a a$, $a A$, and $A A$. Let $u_{i}(x, t)(i=1,2,3)$ be the densities of these three types, and $w=u_{1}+u_{2}+u_{3}$ the total density. For simplicity of the following argument, we suppose that $w$ is constant in $x$ and $t$ (this constant can be thought of as the carrying capacity of the population, assumed independent of the composition $u_{i}$ ). This assumption is not necessary, however; see remarks below.

Our formulation of the dynamical equations for the $u_{i}$ is a generalization of that of Aronson and Weinberger (1975). With $\mathbf{u}=\left(u_{1}, u_{2}, u_{3}\right)$, we let $r(\mathbf{u})$ be the birth rates for the population as a whole (by assumption of constant size, it is also the death rate), $\beta_{i}(\mathrm{u})$ the fractions of the three genotypes among the total births, and $\delta_{i}(\mathbf{u})$ the fractions among the total deaths.

So if $D$ is the spatial diffusivity, the three dynamical equations are

$$
u_{i, t}-D u_{i, x x}=w r\left(\beta_{i}-\delta_{i}\right), \quad i=1,2,3 .
$$

Dividing by $w$, we get similar equations for the "frequencies" $v_{i}=u_{i} / w$. The frequency of allele $a$ in the total population is given by $p=v_{1}+\frac{1}{2} v_{2}$. For it, we have the equation

$$
p_{t}-D p_{x x}=r\left(\beta_{1}+\frac{1}{2} \beta_{2}-\delta_{1}-\frac{1}{2} \delta_{2}\right) .
$$

In the absence of any natural selection, we would have $\delta_{i}=v_{i}, \beta_{1}=p^{2}$, $\beta_{2}=2 p(1-p), \beta_{3}=(1-p)^{2}$. These are the proportions resulting from random mating, equal fecundities, and equal death rates. But we assume weak selection as follows:

$$
\begin{array}{ll}
\delta_{i}=v_{i}\left(1+\varepsilon \gamma_{i}(\mathrm{v})\right), & \beta_{1}=p^{2}\left(1+\varepsilon \eta_{1}(\mathrm{v})\right), \\
& \beta_{2}=2 p(1-p)\left(1+\varepsilon \eta_{2}(\mathrm{v})\right), \\
& \beta_{3}=(1-p)^{2}\left(1+\varepsilon \eta_{3}(\mathrm{v})\right),
\end{array}
$$

for some functions $\gamma_{i}, \eta_{i}$. Necessarily

$$
p^{2} \eta_{1}+2 p(1-p) \eta_{2}+(1-p)^{2} \eta_{3}=v_{1} \gamma_{1}+v_{2} \gamma_{2}+v_{3} \gamma_{3}=0
$$

Then (6.14) becomes

$$
p_{t}-D p_{x x}=\varepsilon \bar{h}(\mathbf{v})
$$


and (6.13) with $i=2$ becomes (setting $v=v_{2}$ )

$$
v_{t}-D v_{x x}=r(v)(2 p(1-p)-v)+\varepsilon \bar{k}(v)
$$

where $\bar{h}$ and $\bar{k}$ are functions of $\mathbf{v}$.

Now we nondimensionalize by letting $r_{0}$ be some reference rate (such as the maximum of $r(\mathrm{v}))$, and by defining $\tau=\varepsilon r_{0} t, \xi=\sqrt{\varepsilon r_{0} / D} x, \rho(\mathrm{v})=r(\mathrm{v}) / r_{0}$. Then $(6.16,6.17)$ become

$$
\begin{gathered}
p_{\tau}-p_{\xi \xi}=h(\mathbf{v}), \\
\varepsilon\left(v_{\tau}-v_{\xi \xi}\right)=\rho(\mathrm{v})(2 p(1-p)-v)+\varepsilon k(v) .
\end{gathered}
$$

This is our basic system of equations. If we discard the assumption that $w$ is constant, then a third equation (for $w$ ) must be added.

As a formal approximation, we set $\varepsilon=0$ to obtain $v=2 p(1-p)$. It follows that $v_{1}=p^{2}, v_{3}=(1-p)^{2}$, or in other words $\mathrm{v}=\mathrm{V}(p)$ (so-called Hardy-Weinberg proportions). Letting $f(p)=h(\mathrm{~V}(p))$, we obtain the single equation

$$
p_{\tau}-p_{\xi \xi}=f(p)
$$

This is like the equation of Fisher considered in $\$ 3$ (though he used a special case). It can be checked that $f(0)=f(1)=0$, and that $f$ may or may not have other zeros in $(0,1)$, depending on the relative magnitudes of

$$
\frac{\eta_{1}(\mathbf{V}(p))-\gamma_{1}(\mathbf{V}(p))}{1-p}, \quad \eta_{2}-\gamma_{2} \text { and } \frac{\eta_{3}-\gamma_{3}}{p} \text {. }
$$

In some cases, for example, (6.20) will have stable wave front solutions. Let us assume it does. It may then be asked, since (6.20) is a formal approximation to the pair of equations (6.18) and (6.19) for small $\varepsilon$, whether the latter pair will then also have a wave front solution which approximates the former in some sense. Suppose $f$ has three simple zeros, as in \$3, so that (6.20) supports a stable front. C. Conley's methods provide a conceptually simple proof of this front's existence, and have the advantage that this proof can be readily extended (Conley, unpublished) to yield the existence of a front solution of (6.18) and (6.19).

7. Pulses. A pulse is a traveling structure whose profile approaches the same limit at $\pm \infty$. Thus, as distinguished from a front, the reacting medium returns to its original state after a pulse traverses it. Signals propagating along a nerve axon are very successfully modeled by pulse solutions of the HodgkinHuxley (HH) or FitzHugh-Nagumo (FHN) systems of reaction-diffusion equations, and this, in fact, is the context within which almost all the work on reaction-diffusion pulses has been performed. Excellent reviews of this work are available (Rinzel (1978), Scott (1975), Hastings (1975), H. Cohen (1971)), and so my comments here will be very brief.

Formally speaking, the HH system is a system of the type (4.1) with $m=4$, and all elements of $D$ zero except the upper left. The FHN system was devised as a simpler approximation to the $\mathrm{HH}$ system, and has $m=2$, again with only one element of $D$ nonzero. Finally, a third system, suggested by McKean (1970), has played an important role in modeling signal propagation. 
It is a modification of the FHN system, in that the nonlinear function appearing in the latter is replaced by a piecewise linear one. This was done with the idea that the resulting system should (1) be tractable analytically, and (2) have solutions with the same qualitative properties as the FHN system.

Both hopes have turned out to be justified for the McKean system; pulse solutions of it have been demonstrated, and their stability analyzed (Rinzel and Keller (1973), Rinzel (1975), Feroe (1978)). Moreover, the qualitative properties of the solutions, established analytically, agreed with those for the HH and FHN equations, which had been obtained by others numerically. The stability analysis in Rinzel and Keller (1973) was according to the linearized criterion, and shows that some of the constructed pulses (the slow ones) are unstable. Feroe (1978), building on the work of Evans (1972), (1975), has recently established rigorously the stability of other pulses (the fast ones).

In addition, a family of wave trains was also constructed by Rinzel and Keller. Again by use of linear stability criteria, some members of the family were judged to be unstable, others probably stable.

The existence of pulse solutions for the HH and/or FHN systems, under various restrictions on the parameters of the equations, has been proved by Carpenter (1976, 1977b), Hastings (1976a, b), and Conley (1975). As is the case for the McKean simplification, there exist two types of pulses: slow ones, thought to be unstable, and fast ones, thought to be stable. Establishing the stability properties of the pulses has proven to be a more difficult task even than their existence, and to date it cannot be said that a completely rigorous proof of stability of pulses for the $\mathrm{HH}$ and FHN equations has been given. Nevertheless, Evans $(1972,1975)$ has come a long way; he has reduced the problem to another criterion, which should be capable of verification, at least by use of a computer. At this point there is strong evidence in favor of stability for the fast pulse.

Carpenter (1976, 1977a, 1977b), Conley (1975a, b), and Hastings (1974, 1976) have also proved the existence of wave trains for the FHN equation, and Carpenter for the $\mathrm{HH}$ equation. In fact, Carpenter also obtained finite wave trains, or $N$-pulse solutions.

Bell and Cook (1977) devised, on physiological grounds, an alternate system of Hodgkin-Huxley type with $m=6$, and proved the existence of pulses and wave trains using the methods of Carpenter.

Singular perturbation techniques have been applied to the FHN system when one of the parameters is small by Casten, Cohen, and Lagerstrom (1975). They construct pulses and wave trains of "traveling plateau" type, with abrupt transitions at the front and back of each pulse. Another conceptual procedure for constructing pulse solutions of certain reaction-diffusion systems by a singular perturbation analysis was brought out by Feinn and Ortoleva (1977). Their solutions have an abrupt transition at only the leading edge.

A criterion for the existence of bifurcating pulse solutions of general reaction-diffusion systems was given by Larson (1977a), but he determined that those he constructed are unstable. 
8. Targets and spirals; slow modulation. Target patterns and spiral configurations for chemically reacting and diffusing media are probably the best documented experimentally of all the possible nontrivial SAS's (Zaikin and Zhabotinsky (1970), Winfree (1972)). Yet their mathematical analysis is very incomplete compared with that of the structures we have considered up to now in this survey.

Away from their centers, these patterns appear locally as plane wave trains, and this suggests the need for a theory of "slowly varying waves." Such a theory, also capable of describing wave trains of other shapes besides circular and planar, was laid down and carefully developed up to a point by Howard and Kopell $(1974,1977)$. Their theory begins with an assumed knowledge of some one-parameter family of plane wave trains and its dispersion relation; out of these ingredients, by means of a formal asymptotic analysis using two length and time scales, solutions are constructed which look locally like plane wave trains, but globally can be patterned in a great variety of ways.

Ortoleva and Ross (1974) also gave a perturbation theory, somewhat different from the one described above, and capable of describing target patterns. In fact if the derivative of the dispersion relation satisfies a certain inequality, their analysis will yield (formally) an entire diverging target pattern, including the center as well. Greenberg $(1976,1978)$ made a similar observation, and also indicated an asymptotic approach to the analysis of rotating spiral patterns. Howard and Kopell (1977) observed that this inequality is unrealistic for the laboratory reaction producing such patterns, so does not necessarily describe a phenomenon which has been observed.

In the opposite case, when the inequality is reversed, Howard and Kopell analyzed the phenomenon of impinging wave trains, which are analogous to converging target patterns. In this case, they also have some results on the very difficult question of the existence of expanding target patterns for $\lambda-\omega$ systems (in preparation).

Spiral patterns appear to be an important SAS for equations describing excitable media. This is evident from numerical computations performed by Winfree (1974), using the McKean model system (see \$7) with diffusion in both equations. An extremely simple cellular three-state model of an excitable medium with interactions between neighboring cells was studied by Greenberg and Hastings (1978), and shown to exhibit spiral patterns. See also (Greenberg, Hassard, and Hastings (1978)). In a model with this simplicity, one is also able to gain insight into the reason for the appearance of spirals. ${ }^{2}$

"Scroll" patterns, three-dimensional versions of spirals, evidently also form an important class of SAS's for excitable media (Winfree (1973)), but any mathematical analysis of them that exists is primitive.

A different type of slow modulation phenomenon was explored by Cohen, Hoppensteadt, and Miura (1977). Rather than having plane wave trains on short space-time scales which are modulated on larger time or space scales, they study homogeneous oscillations whose large-scale modulation itself is a plane wave. Several possible applications are mentioned.

2

The existence of spiral patterns for $\lambda-\omega$ systems has recently been proved by D. S. Cohen, J. C. Neu, and R. R. Rosales, SIAM J. Appl. Math. (to appear). 
9. Close relatives of reaction-diffusion equations.

(a) Discrete versions. Spatial migration and reaction processes can be modeled in other ways besides reaction-diffusion equations (1.1). In population genetics and ecology, populations are often thought of as compartmentalized in space, and spatial migration represented by random transport from one compartment or colony to nearby ones (for example, see Levin (1977)). Spatially compartmentalized models are also used in developmental biology. In fact, Turing (1953) spoke of cell-to-cell as well as continuous diffusion in his pioneering work on cell differentiation.

These models result in high-order systems of ordinary differential equations in place of (1.1). But time is also of ten discretized. In population genetics and ecology, generations or seasons form natural time units for this purpose. It seems likely that most of the results mentioned in this paper for reaction-diffusion systems have valid analogs for the corresponding evolution finite difference equations, in which space and/or time is discretized.

(b) Integrodifference equations. The natural selection model referred to in \$6(d) (see also Aronson and Weinberger (1975)) can be altered by supposing that migration, reproduction, and population control occur in separate yearly seasons. This is carried out, and the resulting model analyzed in Weinberger (1978).

If one assumes that mating is random, then this alternative has the analytically advantageous property that Hardy-Weinberg proportions are maintained at all times. As a result, the system of equations for the three genotypes may be strictly reduced to a single equation for the frequency of the allele $a$. Since time is discrete, the migration of individuals during the course of a year can be represented by a convolution integral over space. The result is an integrodifference equation in $x$ and $t$ for a single dependent variable $u$. (Discrete time models such as this, but without continuous spatial migration, are common in population genetics.) Weinberger (1978) established, for this equation, many of the techniques and results for Fisher's nonlinear diffusion equation, including an asymptotic speed of propagation and the existence of wave fronts.

(c) Integrodifferential equations in neurodynamics and epidemiology. Models of large-scale neural activity have been formulated in recent years by Wilson and Cowan (1973) and others (see Cowan (1974)). A typical example is the pair of equations

$$
\begin{aligned}
& \frac{\partial u}{\partial t}=-u+F\left(u, K_{11} * u-K_{12} * v\right), \\
& \frac{\partial v}{\partial t}=-v+G\left(v, K_{21} * u-K_{22} * v\right),
\end{aligned}
$$

where " $*$ " denotes spatial convolution, and $K_{i j}, F$, and $G$ are given functions. (Here $u$ and $v$ might represent firing intensities of excitatory and inhibitory neurons in a cortex.)

Numerical studies of such models reveal wavelike activity characteristic of reaction-diffusion equations.

Similar models in one and two spatial dimensions have been studied by Ermentrout and Cowan (1977) and the existence of stationary spatial patterns 
demonstrated by bifurcation arguments (as in $\$ 4$ ).

In modeling the geographical spread of a contagious disease, Kendall (1965) proposed a system of three integrodifferential equations for the densities of susceptible, infected, and removed individuals in the population, as functions of space and time. Denote these densities by $u, v$, and $w$; then the equations are

$$
u_{t}=-a u(K * v), \quad v_{t}=a u(K * v)-b v, \quad w_{t}=b v,
$$

where $K$ is a nonnegative averaging kernel. Wave fronts for the system have been studied by Atkinson and Reuter (1976) and others. The situation is similar to that with Fisher's original equation (\$3): $v \equiv 0$ is an unstable rest state; under certain circumstances, there exists a critical speed $c^{*}>0$ such that for each $c>c^{*}$, a wave front exists with velocity $c$. Aronson (1977b) has shown $c^{*}$ to be the asymptotic speed of propagation of an infection which is initially introduced at one location (or more generally, is initially absent for $\left.x>x_{0}\right)$.

More recently, Diekmann (1977) proved the existence of wave fronts for an integrodifferential model based on an epidemic model by Kermack and McKendrick, predating and more general than that of Kendall.

Kendall (1965) also approximated his model by a system of partial differential equations, though not of reaction-diffusion type as we have defined it. He found that wave fronts exist for this model. See Aronson (1977a) for results on this system.

\section{REFERENCES}

H. Amann, 1978, Invariant sets and existence theorems for semilinear parabolic and elliptic systems, J. Math. Anal. Appl. (to appear)

R. Aris, 1975, The mathematical theory of diffusion and reaction in permeable catalysts. I, II, Clarendon Press, Oxford.

D. G. Aronson, 1977a, Topics in nonlinear diffusion, CBMS/NSF Lecture Notes, SIAM (to appear).

1977b, The asymptotic speed of propagation of a simple epidemic, Nonlinear Diffusion, Research Notes in Math., No. 14, Pitman Publishing Co., London, 1-23.

D. G. Aronson and H. F. Weinberger, 1975, Nonlinear diffusion in population genetics, combustion, and nerve propagation, Proc. Tulane Program in Partial Differential Equations and Related Topics, Lecture Notes in Math., vol. 446, Springer, Berlin.

Math. (to appear).

1978, Multidimensional nonlinear diffusion arising in population genetics, Advances in

C. Atkinson and G. E. H. Reuter, 1976, Deterministic epidemic waves, Math. Proc. Cambridge Philos. Soc. 80, 315-330.

J. F. G. Auchmuty, 1978, Qualitative effects of diffusion in chemical systems, Some Mathematical Questions in Biology, Lectures on Math. in the Life Sciences, Amer. Math. Society (to appear).

C. Bardos and J. Smoller, 1978, Instabilité des solutions stationnaires pour des systèmes de reaction-diffusion, C.R. Acad. Sci. Paris Sér. A-B (to appear).

J. Bell and L. P. Cook, 1977, On the solutions of a nerve conduction equation (preprint).

G. Carpenter, 1976, A mathematical analysis of excitable membrane phenomena, Proc. Third Europ. Meeting on Cybernetics and Systems Res.

, 1977a, Periodic solutions of nerve impulse equations, J. Math. Anal. Appl. 58, 152-173.

, 1977b, A geometric approach to singular perturbation problems with applications to nerve impulse equations, J. Differential Equations 23.

R. Casten, H. Cohen, and P. Lagerstrom, 1975, Perturbation analysis of an approximation to Hodgkin-Huxley theory, Quart Appl. Math.32, 365-402. 
N. Chafee, 1974, A stability analysis for a semilinear parabolic partial differential equation, J. Differential Equations 15, 522-540.

K.-N. Chueh, 1975, On the asymptotic behavior of solutions of semilinear parabolic partial differential equations, Ph.D. Thesis, Univ. of Wisconsin.

D. S. Cohen, F. C. Hoppensteadt, and R. M. Miura, 1977, Slowty-modulated oscillations in nonlinear diffusion processes, SIAM J. Appl. Math. 33, 217-229.

H. Cohen, 1971, Nonlinear diffusion problems, Studies in Applied Math. 7, Math. Assoc. of America and Prentice Hall, 27-63.

C. Conley, 1975a, On traveling wave solutions of nonlinear diffusion equations, Dynamic Systems Theory and Appl. (J. Moser, Editor), Lecture Notes in Physics, vol. 38, Springer-Verlag, Berlin and New York.

(preprint).

1975b, On the existence of bounded progressive wave solutions of the Nagumo equation

(to appear).

1978, Isolated invariant sets and the Morse index, CBMS/NSF Lecture Notes, SIAM

C. C. Conley and J. A. Smoller, 1976, Remarks on travelling wave solutions of nonlinear diffusion equations, Structural Stability, The Theory of Catastrophes, and Applications in the Sciences (P. Hilton, Editor), Lecture Notes in Math., vol. 525, Springer-Verlag, Berlin and New York.

E. Conway, D. Hoff, and J. Smoller, 1978, Large time behavior of solutions of systems of nonlinear reaction-diffusion equations, SIAM J. Appl. Math. (to appear).

J. Cowan, 1974, Mathematical models of large-scale nervous activity, Some Mathematical Questions in Biology. V, Lectures on Math. in the Life Sciences, vol. 6, Amer. Math. Soc., Providence, R.I., pp. 101-133.

M. G. Crandall and P. H. Rabinowitz, 1978, The Hopf bifurcation theorem, Arch. Rational Mech. Anal. (to appear).

O. Diekmann, 1977, Threshholds and travelling waves for the geographical spread of infection, Mathematical Centre Report TW 166, Amsterdam.

O. Diekmann and N. M. Temme, 1976, Nonlinear diffusion problemsMathematisch Centrum, Amsterdam.

G. B. Ermentrout and J. D. Cowan, 1977, Large scale activity in neural nets (preprint).

J. W. Evans, 1972, Nerve axon equations. III. Stability of the nerve impulse, Indiana Univ. Math. J. 22, 577-593.

Math. J. 24, 1169-1190.

1975, Nerve axon equations. IV, The stable and the unstable impulse, Indiana Univ.

D. Feinn and P. Ortoleva, 1977, Catastrophe and propagation in chemical reactions, J. Chem. Phys. 67, 2119.

J. Feroe, 1978, Temporal stability of solitary impulse solutions of a nerve equation, Biophys. J. (to appear).

P. C. Fife, 1976a, Boundary and interior transition layer phenomena for pairs of second-order differential equations, J. Math. Anal. Appl. 54, 497-521.

, 1976b, Singular perturbation and wave front techniques in reaction-diffusion problems, Proc. AMS-SIAM Symposium on Asymptotic Methods and Singular Perturbations, New York.

, 1977a, Stationary patterns for reaction-diffusion equations, Nonlinear Diffusion, Proc.

of NSF-CBMS Regional Conference on Nonlinnear Diffusion, Research Notes in Math., No. 14, Pitman, London, pp. 81-121.

358-362. 1977b, On modeling pattern formation by activator-inhibitor systems, J. Math. Biol. 4, 7, $389-415$.

1977c, Asymptotic analysis of reaction-diffusion wave fronts, Rocky Mountain J. Math.

P. Fife and J. B. McLeod, 1977, The approach of solutions of nonlinear diffusion equations to travelling front solutions, Arch. Rational Mech. Anal. 65, 335-361. Also: Bull. Amer. Math. Soc. 81 (1975), 1075-1078.

R. A. Fisher, 1937, The wave of advance of advantageous genes, Ann. of Eugenics 7, 355-369.

A. Gierer and H. Meinhardt, 1972, A theory of biological pattern formation, Kybernetika (Prague) 12, 30-39.

, 1974, Biological pattern formation involving lateral inhibition, Lectures on Math. in the

Life Sciences, vol. 7, Amer. Math. Soc., Providence, R.I., pp. 163-183. 
J. I. Gmitro and L. E. Scriven, 1966, A physicochemical basis for pattern and rhythm, Intracellular Transport (K. B. Warren, Editor), Academic Press, New York and London.

J. M. Greenberg, 1976, Periodic solutions to reaction-diffusion equations, SIAM J. Appl. Math. 30, 199-205.

, 1978, Axi-symmetric, time-periodic solutions to $\lambda-\omega$ systems (to appear).

J. M. Greenberg and S. P. Hastings, 1978, Spatial patterns for discrete models of diffusion in excitable media, SIAM J. Appl. Math. (to appear).

J. M. Greenberg, B. Hassard, and S. P. Hastings, 1978, Pattern formation and periodic structures in systems modeled by reaction-diffusion equations, Bull. Amer. Math. Soc. (to appear).

S. P. Hastings, 1974, The existence of periodic solutions to Nagumo's equation, Quart. J. Math. Oxford Ser. 25, 369-378.

881-895. , 1975, Some mathematical problems from neurobiology, Amer. Math. Monthly 82,

, 1976a, On the existence of homoclinic and periodic orbits for the FitzHugh-Nagumo equations, Quart. J. Math. Oxford Ser. 27, 123-134.

, 1976b, On travelling wave solutions of the Hodgkin-Huxley equations, Arch. Rational Mech. Anal. 60, 229-257.

D. Henry, 1978, Geometric theory of semilinear parabolic equations, Lecture Notes in Math., Springer-Verlag (to appear).

H. Herschkowitz-Kaufman and G. Nicolis, 1972, Localized spatial structures and nonlinear chemical waves in dissipative systems, J. Chem. Phys. 56, 1890-1895.

F. Hoppensteadt, 1975, Mathematical theories of populations: demographics, genetics, and epidemics, Regional Conference Series in Applied Mathematics, No. 20, Society for Industrial and Applied Mathematics, Philadelphia.

L. N. Howard and N. Kopell, 1974, Wave trains, shock fronts, and transition layers in reaction-diffusion equations, SIAM-AMS Proceedings, vol. 8, Providence, R.I., pp. 1-12.

, 1977, Slowly varying waves and shock structures in reaction-diffusion equations, Studies in Appl. Math. 56, 95-145.

D. D. Joseph, 1976, Stability of fluid motions. I, II, Springer-Verlag, New York and Berlin.

Ya. I. Kanel', 1962, On the stabilization of solutions of the Cauchy problem for the equations arising in the theory of combustion, Mat. Sb. 59, 245-288.

, 1964, On the stabilization of the solutions of the equations of combustion theory with initial data of compact support, Mat. Sb. 65, 398-413.

Y. Kametaka, 1976, On the nonlinear diffusion equation of Kolmogorov-Petrouskil-Piskunov type, Osaka J. Math. 13, 11-66.

J. P. Keener, 1978, Activators and inhibitors in pattern formation, Studies in Appl. Math. (to appear).

D. G. Kendall, 1965, Mathematical models of the spread of infections, Mathematics and Computer Science in Biology and Medicine, Medical Research Council.

H. Kielhöfer, 1976, On the Lyapunov-stability of stationary solutions of semilinear parabolic differential equations, J. Differential Equations 22, 193-208.

A. N. Kolmogorov, I. G. Petrovskil, and N. S. Piskunov, 1937, A study of the equation of diffusion with increase in the quantity of matter, and its application to a biological problem, Bjul. Moskovskovo Gos. Univ. 1, no. 7, 1-72.

N. Kopell and L. N. Howard, 1973, Plane wave solutions to reaction-diffusion equations, Studies in Appl. Math. 52, 291-328.

H. Kurland, 1978, Dissertation, University of Wisconsin.

D. A. Larson, 1977a, On the existence and stability of bifurcated solitary wave solutions to nonlinear diffusion equations, J. Math. Anal. Appl.

, 1977b, On models for two-dimensional spatially structured chemo-diffusional signal propagation (preprint).

S. A. Levin, 1977, Population models and community structure in heterogeneous environments (S. A. Levin, Editor), MAA Study in Mathematical Biology, Vol. II: Populations and Communities (to appear).

H. P. McKean, 1970, Nagumo's equation, Advances in Math. 4, $209-223$.

1975, Application of Brownian motion to the equation of Kolmogorov-PetrovskilPiskunov, Comm. Pure Appl. Math. 28, 323-331. 
J. Nagumo, S. Arimoto, and S. Yoshizawa, 1962, An active pulse transmission line simulating nerve axon, Proc. Inst. Radio Engrs. 50, 2061-2070.

G. Nicolis, 1974, Patterns of spatio-temporal organization in chemical and biochemical kinetics, SIAM-AMS Proc. vol. 8, Providence, R.I., pp. 33-58.

P. Ortoleva and J. Ross, 1974, On a variety of wave phenomena in chemical oscillations, J. Chem. Phys. 60, 5090-5107.

1976, Theory of propagation of discontinuities in kinetic systems with multiple time scales: fronts, front multiplicity, and pulses, J. Chem. Phys. 63, 3398-3408.

I. Prigogine and G. Nicolis, 1967, On symmetry-breaking instabilities in dissipative systems, J. Chem. Phys. 46, 3542-3550.

J. Rinzel, 1975, Neutrally stable traveling wave solutions of nerve conduction equations, J. Math. Biol. 2, 205-217.

1978, Integration and propagation of neuroelectric signals, MAA Study in Mathematical

Biology (S. A. Levin, Editor), Math. Association of America (to appear).

J. Rinzel and J. B. Keller, 1973, Traveling wave solutions of a merve conduction equation, Biophys. J. 13, 1313-1337.

F. Rothe, 1975, Über das asymptotische Verhalten der Lösungen einer nichtlinearen parabolischen Differentialgleichung aus der Populationsgenetik, Dissertation, University of Tübingen. , 1978, Convergence to travelling fronts in semilinear parabolic equations (to appear).

D. Sattinger, 1976a, On the stability of waves of nonlinear parabolic systems, Advances in Math. 22, 312-355.

, 1976b, Stability of traveling waves of nonlinear parabolic equations, Proc. of VIIth Inter.

Conf. on Nonlinear Oscillations, East Berlin, 1975.

130-144.

, 1977a, Weighted norms for the stability of traveling waves, J. Differential Equations 25,

1978b, Group theoretic aspects of pattern formation (to appear).

, 1978c, Selection rules for pattern formation (to appear).

A. C. Scott, 1975, The electrophysics of a nerve fiber, Rev. Modern Phys. 47, 487-533. , 1977, Neurophysics, Wiley-Interscience, New York.

L. A. Segel and J. L. Jackson, 1972, Dissipative structure: an explanation and an ecological example, J. Theoret. Biol. 37, 545-559.

L. A. Segel and S. A. Levin, 1976, Application of nonlinear stability theory to the study of the effects of diffusion on predator-prey interactions, Topics in Statistical Mechanics and Biophysics: A Memorial to J. L. Jackson, AIP Conference Proceedings, No. 27.

A. N. Stokes, 1976, On two types of moving front in quasilinear diffusion, Math. Biosci. 31, 307-315.

A. M. Turing, 1953, The chemical basis of morphogenesis, Philos. Trans. Roy. Soc. London Ser. B 237, 37-72.

K. Uchiyama, 1977, The behavior of solutions of some non-linear diffusion equations for large time (preprint).

W. Wasow, 1965, Asymptotic expansions for ordinary differential equations, Interscience, New York.

H. F. Weinberger, 1978, Asymptotic behavior of a model in population genetics, Springer Lecture Notes, Indiana Univ. Seminar in Applied Mathematics, ed. J. Chadam (to appear).

H. R. Wilson and J. D. Cowan, 1973, A mathematical theory of the functional dynamics of cortical and thalamic nervous tissue, Kybernetika (Prague) 13, 55-80.

A. T. Winfree, 1972, Spiral waves of chemical activity, Science 175, 634-636.

$937-939$.

1973, Scroll-shaped waves of chemical activity in three dimensions, Science 181,

1974, Rotating solutions of reaction-diffusion equations in simply-connected media,

SIAM-AMS Proceedings, vol. 8, Providence, R.I., pp. 13-31.

A. N. Zaikin and A. M. Zhabotinsky, 1970, Concentration wave propagation in two-dimensional liquid-phase self-oscillating system, Nature 225, 535-537.

Ya. B. Zel'dovic, 1948, On the theory of propagating flames, $\dot{Z}$. Fiz. Him. 22, $27-48$.

Department of Mathematics, Universtty of ARizona, Tucson, Arizona 85721 